\title{
Die ökonomische Erforschung der lateinamerikanischen «Mittelklassen»: Ein Review aus klassentheoretischer Perspektive
}

\author{
Matthias SCHULZ1 und Bahareh GONDANI ${ }^{2}$ \\ Universität Fribourg
}

\begin{abstract}
Die in den letzten Jahren erschienenen internationalen entwicklungsökonomischen Publikationen zu lateinamerikanischen "Mittelklassen» erfahren eine grosse wissenschaftliche und öffentliche Aufmerksamkeit. Obwohl die in den ökonomischen Publikationen angewendeten Konzeptualisierungen von "Mittelklassen» deutlich von jenen der sozialwissenschaftlichen Forschung abweichen, werden die Ergebnisse der ökonomischen Forschung in viele sozialwissenschaftlichen Publikationen referenziert. Um die Frage der Anknüpfbarkeit der ökonomischen und sozialwissenschaftlichen Forschungsstränge zu klären, diskutiert der vorliegende Artikel die Ergebnisse der ökonomischen Forschung zu lateinamerikanischen «Mittelklassen» vor dem Hintergrund der verwendeten Konzepte von Klasse und den Operationalisierungen ihrer Mitten. Das Review basiert auf einer systematischen Publikationssuche der wirtschaftswissenschaftlichen Veröffentlichungen zum Thema im Beobachtungszeitraum 2000-2018.
\end{abstract}

Schlüsselwörter: Mittelklasse, Entwicklungsökonomie, Klassenanalyse, Lateinamerika, Ungleichheit, wissenschaftliches Review

\section{Einleitung ${ }^{3}$}

Strukturelle soziale Ungleichheit und soziale Klassen sind seit jeher Themen der Gesellschaftswissenschaften, wenngleich sie seit den 1980er Jahren in Lateinamerika ihren zentralen Stellenwert in Soziologie und Politikwissenschaften in Folge der gesellschaftspolitischen Ereignisse, mit denen sich Lateinamerika in dieser Zeit konfrontiert sah, eingebüsst hatten. Die Überwindung repressiver Militärdiktaturen, die Staatsschuldenkrisen und daraufhin implementierten marktliberalen Strukturreformen, von denen viele lateinamerikanische Länder betroffen waren, hatten zur Konsequenz, dass Fragen institutioneller, soziopolitischer und

\footnotetext{
${ }^{1}$ Dipl.-Soz. Matthias Schulz ist Diplomassistent am Departement of Social Work, Social Policy and Global Development der Universität Fribourg (CH) (matthias.schulz@unifr.ch).

${ }^{2}$ Dipl.-Soz. Bahareh Gondani ist Doktorandin am Departement für Sozialarbeit, Sozialpolitik und globale Entwicklung der Universität Fribourg (CH) (email@gondani.net).

${ }^{3}$ Wir danken Prof. Dr. Monica Budowski und Dr. Eveline Odermatt für die zahlreichen Kommentare und vielen Gespräche, die die Qualität dieses Artikels erheblich gesteigert haben.
}

DOI: http://dx.doi.org/10.18753/2297-8224-133 
zivilgesellschaftlicher Bedingungen einer Re-Demokratisierung und zum anderen die Effekte von Wirtschaftskrisen und Strukturreformen auf Armutslagen ins Zentrum des wissenschaftlichen Interesses rückten (vgl. Filgueira 2001; Espinoza 2002; Franco/Hopenhayn 2010; Solís/Boado 2016). Um die Jahrtausendwende richtet sich die wissenschaftliche Aufmerksamkeit zunehmend auf über Armut hinausgehende sozialstrukturelle und ungleichheitsrelevante Folgen der Strukturreformen (Filgueira 2001; Atria 2004; Boris 2008; Wehr 2011). Insbesondere interessieren sich neben den Gesellschaftswissenschaften nun auch die Wirtschaftswissenschaften für diese Themen und prägen stark die internationale wissenschaftliche Debatte. Die Publikationen greifen damit ein bis dahin von den Wirtschaftswissenschaften weitgehend vernachlässigtes Thema auf, heben es auf die Agenda der grossen entwicklungspolitischen Organisationen und bringen es in den öffentlichen Diskurs ein. Anders als von den Reformer_innen erwartet, war Lateinamerika gegen Ende der 1990er Jahre die Region der Welt mit der grössten Ungleichheit und zeichnete sich durch ausserordentlich geringe Mobilität und vergleichsweise geringem Wirtschaftswachstum aus (Behrman/Gaviria/Székely 2001; Ferranti/Perry/Ferreira/Walton 2004; Torche 2005). Vor dem Hintergrund des ausbleibenden wirtschaftlichen Erfolgs der Reformen, kritisierten seit Ende der 1990er Jahre zunehmend mehr Entwicklungsökonomen die Strukturanpassungsprogramme. Ihnen ging es dabei nicht um eine generelle Abkehr, sondern darum, negative Nebenfolgen der Reformen (Anstieg der Arbeitslosigkeit, Rückgang der Löhne, Zunahme von Armut und Einkommensungleichheit) zu thematisieren, ihre Ursachen zu verstehen und flankierende Massnahmen zu entwickeln, damit die zentralen Ziele der Reformen (vor allem Wirtschaftswachstum und wirtschaftliche Stabilität) nicht gefährdet werden. In dieser Debatte rückt die «Mittelklasse» ${ }^{4}$ in den Fokus der wissenschaftlichen Aufmerksamt. Das besondere Interesse an dieser sozialen Gruppe wird häufig zum einen über die besondere Rolle der «Mittelklasse» als Träger von demokratischer und wirtschaftlicher Entwicklung und zum anderen über ihre besondere Betroffenheit von den marktliberalen Strukturreformen der 1980er und 1990er Jahre begründet (u.a. Birdsall/Graham/Pettinato 2000).

Obschon sich sowohl die Sozialwissenschaften als auch die Wirtschaftswissenschaften seit der Jahrtausendwende im besonderen Masse für die lateinamerikanischen «Mittelklassen» interessieren, verlaufen die Diskurse weitestgehend getrennt voneinander. Während die sozialwissenschaftliche «Mittelklasse»-Forschung - vor allem jene über die «neuen Mittelklassen» - durchaus Kenntnis von den wirtschaftswissenschaftlichen Publikationen und Ergebnissen nimmt und diese mitunter zitieren, beziehen sich die Autor_innen der ökonomischen «Mittelklasse»-Forschung nicht auf die Konzepte und Ergebnisse der seit Mitte des 20. Jahrhundert bestehenden sozialwissenschaftlichen Forschung über lateinamerikanische «Mittelklassen». So knüpfen die ökonomischen Konzeptualisierungen von «Mittelklassen» auch nicht an bestehende sozialwissenschaftlich Konzepte an. Beide Disziplinen verwenden zwar den Begriff «Mittelklasse», erfassen mit diesem Begriff aber aufgrund der divergierenden Konzeptualisie-

\footnotetext{
${ }^{4}$ Klassenkategorien, ihre Bezeichnungen und Abgrenzungen sind in der Regel deduktive Klassifikationen sozialer Akteure. Hinter denselben Bezeichnungen (wie bspw. «Mittelklasse») stehen dabei häufig unterschiedliche und z.T. nicht explizierte theoretische Konzeptualisierungen und Operationalisierungen. Um auch textlich hervorzuheben, dass es sich bei diesen Klassifikationen um „papierne Konstruktionen“ (Krais/Gebauer 2002: 37) handelt, sind in diesem Artikel alle Klassenbezeichnungen in Guillemets («») gesetzt.
} 
rungen unterschiedliche Bevölkerungssegmente und kommen daher auch zu unterschiedlichen Ergebnissen. Um die Frage zu klären, ob die ökonomische Befassung mit den lateinamerikanischen «Mittelklassen» aus sozialwissenschaftlicher Perspektive neue Erkenntnisse und Anknüpfungspunkte bietet, zeichnen wir die Entwicklung der ökonomischen Beschäftigung mit den lateinamerikanischen «Mittelklassen» seit der Jahrtausendwende nach und diskutieren ihre z.T. divergenten Ergebnisse und Deutungen vor dem Hintergrund der verwendeten Konzepte von «Klasse» und der Operationalisierungen ihrer Mitten.

Zum methodischen Vorgehen: Es wurde eine systematische Recherche in einschlägigen Literaturdatenbanken ${ }^{5}$ mit zuvor festgelegten spanischen, englischen und deutschen Stichwörtern $^{6}$ sowie einem umfangreichen Reference-Follow-Up durchgeführt. Die über 1100 gefundenen Publikationstitel und zugehörigen Abstracts wurden für den vorliegenden Artikel durch eine scan and skim selection auf jene Titel begrenzt, die sich aus ökonomischer ${ }^{7}$ Perspektive mit der Entwicklung der «Mittelklassen» in Lateinamerika seit dem Millennium beschäftigen (ca. 120) (vgl. nach Jesson/Matheson/Lacey 2011). Die ökonomischen Publikationen sind in der Regel englischsprachig, werden von westlichen Entwicklungsökonomen und grossen internationalen Entwicklungsorganisationen herausgegeben. Sie sind empirisch quantitativ und meist ländervergleichend angelegt. Andere ungleichheitsrelevante Kategorien wie Geschlecht oder Ethnizität und ihre Verwobenheit werden von diesen in der Regel nicht berücksichtigt.

Im Folgenden werden zunächst die unterschiedlichen Konzepte und Operationalisierungen der «Mittelklassen» dargestellt, die in ökonomischen Publikationen zu lateinamerikanischen «Mittelklassen» zur Anwendung kommen. Anschliessend wird der Verlauf der ökonomischen Diskussion nachgezeichnet. Die neunzehn Jahre des Beobachtungszeitraums (20002018) unterteilen wir dabei anhand des thematischen Fokus der Publikationen grob in zwei Phasen. In der ersten Phase - etwa von 2000 bis 2010 - beschäftigen sich die Publikationen mit ungleichheitsrelevanten Effekten der Strukturanpassungsprogramme der 1980er Jahre und fokussieren dabei auf die «Mittelklassen», da sie nach Ansicht der Autor_innen durch diese besonders unter Druck geraten sind. Es handelt sich hierbei um die Publikationstätigkeit einiger weniger Autor_innen, die explizit versuchen das Thema «Mittelklassen» auf die politische Agenda zu heben. In der zweiten Phase - etwa von 2010 bis 2018 - stehen das Wirtschaftswachstum der vorhergehenden Dekade und die Expansion der «Mittelklassen» im Vordergrund. Die lateinamerikanische «Mittelklasse» als Subjekt rückt nun erstmals empirisch in den Blick, indem ihre soziodemografischen Merkmale, politischen Einstellungen und das Ausmass ihrer sozialen Integration in relevanten gesellschaftlichen Institutionen empirisch beforscht werden. Die zweite Phase ist die Hochphase der ökonomischen Beschäftigung mit den «Mittelklassen» Lateinamerikas, in der die meisten Artikel und mehrere umfangrei-

${ }^{5}$ Verwendete Suchportale: googlescholar, scielo, redalyc, CEPAL, CLACSO, CEDLAS, Weltbank.

${ }^{6}$ Verwendete Stichwörter:

Spanisch: clase media, estrato medio, sectores medios, capas medias, desigualdad, movilidad, estratificación.

Englisch: middle class, inequality, stratification, mobility.

Deutsch: Mittelklasse, Mittelschicht, Ungleichheit, Mobilität.

Diese Stichworte wurden jeweils kombiniert mit dem Stichwort Lateinamerika/Latin America/latino america.

${ }^{7}$ An anderer Stelle werden wir die soziologische Befassung mit dem Thema nachzeichnen und diskutieren. 
che und häufig zitierte Sammel- und Sonderbände ${ }^{8}$ entstehen. Der Artikel schliesst mit einer klassentheoretischen Kritik ökonomischer Konzeptualisierungen von «Klassen». Wir werden zeigen, dass beim Import des «Mittelklasse»-Begriffs aus der Soziologie in die ökonomische Forschung wesentliche Elemente verloren gehen. Durch die theoretisch wenig fundierten Konzeptualisierungen von «Klasse» und arbiträren Abgrenzungen ihrer Mitte kommt es zu widersprüchlichen empirischen Ergebnissen, sodass kaum noch klare Aussagen über das eigentliche Untersuchungsobjekt «Mittelklassen» und ihre Entwicklung möglich sind.

\section{Welche Mitte?}

Jede empirische Studie zu «Mittelklassen» basiert zwangsläufig auf impliziten oder expliziten Konzepten und Operationalisierungen des Begriffs «Klasse» und dessen Grenzen. In den ökonomischen Publikationen über lateinamerikanische «Mittelklassen» wird häufig kursorisch auf Philosophen oder Soziologen wie Aristoteles, Marx, Weber, Goldthorpe oder Wright eingegangen. Ein theoretisch begründetes Klassenkonzept oder eine explizite Anknüpfung an eine bestehende Klassentheorie findet sich jedoch an keiner Stelle. Die Frage, was die in der Regel verwendeten Einkommensklassenmodelle abbilden wollen und welche Aussagen überhaupt auf Basis von Einkommensklassenmodellen möglich sind, wird nicht thematisiert. Aus der Art der Verwendung des «Mittelklasse»-Begriffs geht jedoch implizit hervor, dass Einkommensklassen mit dem Anspruch gebildet werden, reale soziale Gruppen abzubilden.

In der Regel wird die Kategorie «Mittelklasse» über den metrischen Indikator Einkommen $^{9}$ operationalisiert. Eine gegebene Einkommensverteilung wird dabei in mehrere Einkommensgruppen unterteilt und damit ein vertikales Einkommensklassenmodell generiert. Dabei werden zumeist im ersten Schritt über Haushaltsbefragungen gewonnene Haushaltseinkommen in Pro-Kopf-Einkommen bzw. Äquivalenzeinkommen umgerechnet. Für internationale Vergleiche wird das Einkommen in Kaufkraftparitäten eines Referenzjahres justiert (PPP). ${ }^{10}$ Im zweiten Schritt werden Einkommensschwellen definiert und die einzelnen Haushalte oder Personen den jeweiligen Einkommensklassen zugeordnet. Je nach Ansatz wird die Einkommensverteilung in unterschiedlich viele Einkommensklassen unterteilt: Häufig erfolgt eine Dreiteilung der Einkommensverteilung in "Arme», «Mittelklasse» und «Oberklasse» ${ }^{11}$. Bei einigen Studien wird die «Mittelklasse» in mehrere Unterkategorien wie «untere Mittelklasse» und «obere Mittelklasse» ausdifferenziert (bspw. Castellani/Parent/Zenteno Gonzalez 2015; Penfold et al. 2014) oder es wird zwischen den «Armen» und der «Mittelklasse» noch

\footnotetext{
${ }^{8}$ Es handelt sich bei diesen um von der OECD, der Weltbank und der Inter-Amerikanischen Entwicklungsbank (IDB) herausgegebene Publikationen (OECD 2010; Grynspan/Paramio 2012; Ferreira/Messina/Rigolini/López-Calva/Lugo 2013; Dayton-Johnson 2015a). Zeitgleich publizierte die Wirtschaftskommission für Lateinamerika und die Karibik (CEPAL) zwei Sammelbände mit hauptsächlich soziologischem Fokus (Bárcena Ibarra/Serra 2010; Franco/Hopenhayn/León 2010).

${ }^{9}$ Je nach Datenlage wird zum Teil nicht auf Haushaltseinkommen, sondern auf Haushaltsausgaben zurückgegriffen.

${ }^{10} \mathrm{Da}$ Haushaltsmitglieder nicht im selben Masse konsumieren und es Synergieeffekte gibt, wird das Haushaltseinkommen in der Regel nicht einfach durch die Summe der Haushaltsmitglieder geteilt, sondern diese gehen mit unterschiedlichem Gewicht in die Berechnung ein. Für die Bestimmung der Gewichte gibt es unterschiedliche Verfahren (vgl. http://www.oecd.org/els/soc/OECD-Note-EquivalenceScales.pdf).

${ }^{11}$ Auch als «affluent», «elite», «rich» bezeichnet.
} 
die Klasse der «Vulnerablen» ${ }^{12}$ (bspw. López-Calva/Ortiz-Juárez 2011; Ferreira et al. 2013) eingeführt. Die Hauptunterschiede dieser Ansätze liegen in der Anzahl der gebildeten Einkommensklassen sowie der jeweiligen Schwellenwerte und deren Begründung. Je nach Wahl dieser Werte kann es sich bei den so definierten «Mittelklassen» um unterschiedliche, sogar disjunkte Bevölkerungssegmente handeln. Tabelle 1 enthält eine Übersicht häufig verwendeter bzw. wichtiger «Mittelklasse»-Definitionen.

Tabelle 1: Häufig verwendete «Mittelklasse»-Definitionen

\begin{tabular}{|c|c|c|}
\hline Art der Definition & Schwellenwerte & Referenz-Publikationen der jeweiligen Definition \\
\hline \multicolumn{3}{|c|}{ Relative Definitionen } \\
\hline Median der & $75 \%<y<125 \%$ & Birdsall/Graham/Pettinato 2000 \\
\hline Einkommensverteilung & $50 \%<y<150 \%$ & OECD 2010 \\
\hline Perzentile der & $\mathrm{P} 20<\mathrm{y}<\mathrm{P} 80\left(\sum 60 \%\right)$ & Easterly 2001 \\
\hline \multirow[t]{3}{*}{ Einkommensverteilung } & $\mathrm{P} 20<\mathrm{y}<\mathrm{P} 90\left(\sum 70 \%\right)$ & Solimano 2008 \\
\hline & $\mathrm{P} 40<\mathrm{y}<\mathrm{P} 90\left(\sum 50 \%\right)$ & Estache/Leipziger 2009b \\
\hline & $\mathrm{P} 70<\mathrm{y}<\mathrm{P} 90\left(\sum 20 \%\right)$ & Huber 2009 \\
\hline \multicolumn{3}{|c|}{ Absolute Definitionen } \\
\hline \multirow{6}{*}{$\begin{array}{l}\text { Absolutes Einkommen } \\
\text { pro Kopf und Tag }\end{array}$} & $\$ 2<y<\$ 10$ & Banerjee/Duflo 2008 \\
\hline & $\$ 2<y<\$ 13$ & Ravallion 2009 \\
\hline & $\$ 2<y<\$ 20$ & Castellani/Parent 2011 \\
\hline & $\$ 12<y<\$ 50$ & Milanovic/Yitzhaki 2002 \\
\hline & $\$ 10<y<\$ 50$ & López-Calva/Ortiz-Juárez 2011; Ferreira et al. 2013 \\
\hline & $\$ 10<y<\$ 100$ & Kharas 2010; Cârdenas/Kharas/Henao 2015 \\
\hline \multirow[t]{2}{*}{ Andere } & $\$ 10<\mathrm{y}<\mathrm{P} 95$ & Birdsall 2010 \\
\hline & $1 \mathrm{xNPL}<\mathrm{y}<3 \mathrm{xNPL}$ & Castellani/Parent 2011 \\
\hline
\end{tabular}

Zur Bestimmung der Schwellenwerte werden in der Regel relative (i und ii) oder absolute (iii) exogene Verfahren ${ }^{13}$ angewendet:

(i) In Median-Definitionen wird die «Mittelklasse» als Einkommensgruppe im Zentrum der Einkommensverteilung konzeptualisiert. Die «Mittelklasse» in diesen Definitionen wird durch prozentuale Abweichungen von Medianeinkommen bestimmt. In Abhängigkeit von Enge oder Breite des definierten Bereichs um den Median und dem Ausmass der Ungleichheit der Einkommensverteilung kann der Anteil der «Mittelklasse» an der Gesamtbevölkerung erheblich variieren. Dieses Verfahren trägt der Erkenntnis Rechnung, dass ab einem bestimmten

\footnotetext{
${ }^{12}$ Bei Birdsall et al. (2014) auch als «Struggler» bezeichnet.

${ }^{13}$ Endogene Verfahren werden in der «Mittelklasse»-Forschung vergleichsweise selten angewendet, weshalb wir in diesem Artikel nicht weiter darauf eingehen. Beispiele für auf endogene Verfahren basierende «Mittelklasse»-Studien: parametrische Definitionen (Olivieri 2007; Zhu 2005), Polarisierungsindizes (Gasparini/Horenstein/Olivieri 2006; Cruces/LópezCalva/Battistón 2011; Neri 2015) oder Selbstzuschreibungen (Lora/Fajardo 2011; 2013; Penfold/Rodríguez Guzmán 2014).
} 
Wohlstandsniveau nicht mehr das absolute, sondern das relative Einkommensniveau als Erklärungsgrund für subjektives Wohlbefinden und andere Indikatoren relevant wird (Easterlin 1974; Birdsall et al. 2000; Pressman 2011). Diese Definitionen sind sensibel für relative Veränderungen der Kurve der Einkommensverteilung, reagieren aber nicht auf eine Verschiebung der Gesamtverteilung (Fahrstuhleffekt). Sie sind daher in der Lage, Informationen zur landesspezifischen Einkommensverteilung und zur Positionierung der mittleren Einkommenskategorien in diesem Gefüge zu liefern.

(ii) Perzentil-Definitionen bestimmen die «Mittelklasse» über Perzentile der Einkommensverteilung. Easterly (2001) definiert das zweite bis vierte Einkommensquintil als «Mittelklasse». Die «Mittelklasse» bildet dadurch - wie bei der Bestimmung durch den Median - die mittlere Einkommenskategorie. Anders als beim Median ist die relative Grösse der Kategorie jedoch per Definition stabil (in diesem Fall 60 \%). Typischerweise interessiert man sich bei derartigen Ansätzen für die Veränderung sozioökonomischer Indikatoren, bspw. für den Einkommensanteil, den diese grössenunveränderliche Kategorie am Gesamteinkommen hat (Cruces et al. 2011). Dadurch können Entwicklungen wie der relative Anstieg der Einkommen der «Mittelklasse» sichtbar werden. Die Wahl der Perzentile variiert je nach Publikation erheblich (vgl. Cruces et al. 2011). So umfasst die «Mittelklasse» bei Solimano (2008; 2009) $70 \%$ und anders als bei Easterly (2001) - liegt sie zwischen Perzentil 20 und Perzentil 90. Im Gegensatz dazu definiert Huber (2009) die «Mittelklasse» als jene 20 \% der Bevölkerung, die zwischen Perzentil 70 und Perzentil 90 liegen.

Relative Definitionen tragen vor allem der Erkenntnis Rechnung, dass ab einem bestimmten Wohlstandsniveau das absolute Einkommensniveau für Akteure nicht mehr relevant ist, sondern das relative Einkommensniveau gegenüber ihrem Umfeld (Easterlin 1974; Sen 1983; Birdsall et al. 2000; Pressman 2011). Vor diesem Hintergrund haben alle relativen Definitionen den Vorteil, dass sie die landesspezifische Einkommensverteilung in Rechnung stellen. Das macht inhaltlich sinnvolle internationale Vergleiche möglich, führt jedoch dazu, dass bei Ländern mit grossen Wohlstandsunterschieden, jemand, der in einem Land zur «Mittelklasse» gehört, in anderen Ländern als arm gelten kann. Unter Umständen werden mit demselben Begriff völlig unterschiedliche soziale Lagen gefasst. Relative Definitionen fokussieren auf die Mitte, verstanden als die Mitte der Einkommensverteilung. In Ländern mit sehr geringen Medianeinkommen können daher Teile der «Mittelklasse» sogar unterhalb der jeweiligen nationalen Armutsschwelle liegen (Castellani/Parent/Zentero 2014). Cruces et al. (2011) zeigen, dass bei gängigen relativen Definitionen $30 \%$ bis $40 \%$ der «Mittelklasse»-Angehörigen in Lateinamerika - gemessen an nationalen Standards - arm sind. Sie betrachten dies als Manko relativer Definitionen, da die «Mittelklasse» in der Regel als jene Klasse verstanden wird, die gerade nicht arm ist und sich durch ein bestimmtes Konsumniveau auszeichnet. Ein weiterer Nachteil relativer Definitionen aus Sicht von Cruces et al. (2011) ist, dass allgemeine Einkommenssteigerungen in der Gesamtgesellschaft empirisch unsichtbar bleiben, weil sich die «Mittelklasse»-Definition mit der Verteilung verschiebt. Nur wenn sich die Verhältnisse zwischen den Bevölkerungsgruppen verändern würden, würde sich auch die «Mittelklasse» in ihrer Grösse verändern. Ferreira et al. (2013) bemerken in ihrer einflussreichen Publikation in 
diesem Zusammenhang völlig richtig, dass aufgrund stabiler Einkommensverteilungen die Grösse der relativ definierten «Mittelklassen» auch bei Wirtschaftswachstum stabil ist.

(iii) Absolute Definitionen bestimmen die «Mittelklasse» über absolute, kaufkraftbereinigte und standardisierte Einkommensniveaus als Schwellenwerte (pro Kopf und Tag). Die meist diskutierten absoluten Ansätze wurden von den Entwicklungsökonomen Milanovic/Yitzhaki 2002 (\$2-\$13), Ravallion 2009 (\$2-\$13), Banerjee/Duflo 2008 (\$2-\$10) und Kharas 2010 (\$10-\$100) zur Berechnung der «globalen Mittelklasse» entwickelt. Die variierenden Grenzwerte werden oft über bereits bestehende nationale Grenzwerte bestimmter Referenzländer begründet. So basiert Ravallions unterer Schwellenwert von \$2 auf dem Durchschnitt der nationalen (absoluten) Armutsschwellen der „developing world“, die auf den jeweiligen lokalen Warenkörben basieren. Der obere Schwellenwert von \$12 entspricht der US-amerikanischen Armutsgrenze (Ravallion 2009). ${ }^{14}$ Die Verwendung absoluter «Mittelklasse»-Definitionen wird damit begründet, dass sie auf einen bestimmten Lebensstandard sowie ein bestimmtes Konsumniveau verweisen, das erst ab einer bestimmten absolut definierten Einkommensschwelle realisierbar sei (Birdsall/Lustig/Meyer 2014). Dieses Konsumniveau wird durch die Globalisierungsprozesse hauptsächlich durch globale Preise bestimmt und daher länderübergreifend definiert. Die Entscheidung für bestimmte internationale absolute Schwellenwerte hat erhebliche Folgen darauf, wie gross die so bestimmte «Mittelklasse» ist und welche Bevölkerungssegmente erfasst werden. Vergleicht man bspw. Ravallions (2009) Definition mit der von López-Calva und Ortiz-Juárez (2011), so gibt es keinerlei Überschneidung der beiden als «Mittelklassen» kategorisierten Aggregate. Die einzige Gemeinsamkeit, die alle Haushalte per Definition teilen, ist, dass sie nicht absolut arm sind. Daher liegen die so definierten «Mittelklassen» keineswegs automatisch in der Mitte der Einkommensverteilung, wie es die Bezeichnung suggeriert und es bei relativen Definitionen auch der Fall ist. Im Gegenteil, die Menschen der so definierten «Mittelklassen» gehören in Lateinamerika zu den oberen Einkommensbezieher_innen (siehe Phase II).

In der nachfolgenden Diskussion von Phase I und II werden wir zeigen, dass die unterschiedlichen Konzeptualisierungen von «Mittelklasse» und die variierenden Schwellenwerte divergierende Ergebnisse und daher auch unterschiedliche Einschätzungen der sozialstrukturellen Entwicklungen in der Region zur Folge haben.

\section{Phase I - Die Krise des Washington Consensus und die Entdeckung der «Mittelklasse» (2000 bis 2010)}

Die «Mittelklasse» als Forschungsgegenstand spielte in ökonomischen Untersuchungen zu Lateinamerika vor der Jahrtausendwende kaum eine Rolle. Dies ändert sich erst, als internationale Entwicklungsökonom_innen beginnen die sozialen und wirtschaftlichen Effekte der in

\footnotetext{
${ }^{14}$ Weitere absolute Definitionen, bei denen nationale Grenzwerte verschiedener Länder als Unter- oder Obergrenze verwendet werden, sind z.B. Brasilien-Italien (Milanovic/Yitzhaki 2002) und Portugal-Luxembourg (Bhalla 2007; Kharas 2010; Cârdenas et al. 2015).
} 
den 1980er Jahren in vielen Ländern Lateinamerikas eingeführten marktliberalen Reformen $\mathrm{zu}$ analysieren.

Die Ölpreisschocks der 1970er Jahre und die einsetzende Weltwirtschaftsrezession führten Anfang der 1980er Jahre zu Staatsschuldenkrisen in vielen lateinamerikanischen Ländern. Die stärksten Probleme der Region waren aus Sicht der Lehrbuchökonomie die hohe Inflation, eine hohe öffentliche Verschuldung, eine unbeständige Geldpolitik sowie zu hohe Wechselkurse und Handelshemmnisse (vgl. Birdsall 2007b). Die daraufhin auf Druck der ausländischen Geldgeber (vor allem der Weltbank und des Internationalen Währungsfonds IWF) implementierten Strukturanpassungsprogramme bedeuteten eine radikale Abkehr vom vorherigen Modell der importsubstituierenden Industrialisierung, die in den meisten Ländern Lateinamerikas die Grundlagen einer relativ grossen und stabilen «Mittelklasse» gebildet hatte (Expansion öffentlicher Beschäftigung, Ausbau sozialer Sicherung, Bildungsexpansion, etc.). Diese Strukturanpassungsprogramme bestanden hauptsächlich aus marktliberalen Reformen, die häufig als Washington Consensus bezeichnet werden (Williamson 1990) und sollten zu Wirtschaftswachstum, makroökonomischer Stabilität und Armutsreduktion führen. Der Rückzug des Staates aus der Wirtschaft, die Liberalisierung des Aussenhandels und die Deregulierung der Märkte hatten massive sozialstrukturelle Folgen: Die Privatisierung öffentlicher Funktionen und Unternehmen, der Wegfall von Subventionen sowie der Konkurrenzdruck durch die Öffnung der Märkte für die häufig günstigeren ausländischen Produkte, dem viele Unternehmen nicht standhalten konnten, hatten grosse Entlassungswellen zur Folge. Kürzungen im öffentlichen und privaten Sektor und die hohe Inflation führten ferner zu einem Rückgang des Reallohnniveaus für jene, die noch eine Beschäftigung hatten. Des Weiteren führte die Flexibilisierung der Arbeitsmärkte durch den Abbau arbeits- und sozialrechtlicher Sicherungen zu einer massiven Zunahme von informellen und prekären Beschäftigungsverhältnissen. Die Folgen waren eine hohe Arbeitslosigkeit, die Zunahme der Einkommensungleichheit und ein erheblicher Anstieg der Armut (Klein/Tokman 2000; Birdsall/La Torre 2001; Portes/Hoffman 2003; Sangmeister 2004; Solimano 2006b; Solimano/Soto 2006; Torche 2006; Boris 2008; Franco/Hopenhayn 2010; Sosa Elízaga 2011). Auch die Erwartungen hinsichtlich der formulierten Ziele der Strukturanpassungsprogramme wurden bis zur Jahrtausendwende nicht erfüllt: Die Wirtschaft der Region wuchs zwar, ihr Wirtschaftswachstum fiel im globalen Vergleich jedoch zurück. Auch die angestrebte wirtschaftliche Stabilität wurde nicht erreicht, da die lateinamerikanischen Ökonomien durch die Liberalisierung des Aussenhandels nun verstärkt externen wirtschaftlichen Schocks und volatilen internationalen Kapitalflüssen ausgesetzt waren. Ferner gingen die infolge der Krise zunächst angestiegenen Armutsquoten zwar zurück, stagnierten dann jedoch bei einem Drittel der Bevölkerung (Alesina/Rodrik 1994; Lustig 1995; Altimir 1996; Psacharopoulos/Morley/Fiszbein/Lee/Wood 1997; IDB 1998; Rodrik 1999; 2001; Morley 2001; Székely/Hilgert 2002; Gasparini 2003; Justino/Litchfield/Whitehead 2003; Székely 2003; Sangmeister 2004; Solimano 2005). In der Folge führte der mangelnde Erfolg der Reformen zu steigendem Unmut in der Bevölkerung und einem Anstieg öffentlicher Proteste gegen die Politik der Weltbank und des IWFs (Birdsall et al. 2000; Birdsall/La Torre 2001; Graham/Sukhtankar 2002; Harris 2000; 2002; Huber 2009).

Vor dem Hintergrund des Ausbleibens des erwarteten Erfolgs der Reformen, der zunehmenden Einkommensungleichheit und des steigenden Unmuts gegen die Reformen erstarkt 
unter internationalen Entwicklungsökonom_innen eine Debatte um Fragen über die Zusammenhänge zwischen wirtschaftlichem Wachstum, Einkommensungleichheit und Armut (Sen 1995; Atkinson 1997; Deininger/Squire 1998; Solimano 1998; Aghion/Caroli/García-Peñalosa 1999; Kanbur/Lustig 1999; Barro 1999b; Birdsall 2001). ${ }^{15}$ Hatte die Mehrheit der Ökonom_innen Einkommensungleichheit bis dahin als essentielle Motivationsstruktur von Gesellschaften oder aber als vernachlässigbare Nebenfolge ökonomischer Prozesse betrachtet, identifizieren die meisten Autor_innen in der nun geführten Debatte die Einkommensungleichheit als bedeutende Ursache für geringeres Wirtschaftswachstum und gesellschaftliche Instabilität.

Im Kontext dieser Diskussion entdecken einige Entwicklungsökonom_innen um die Jahrtausendwende die «Mittelklasse». Die herausragenden und auch später am häufigsten zitierten Autor_innen dieser ersten Generation sind William Easterly, Branko Milanovic, Shlomo Yitzhaki, Nancy Birdsall, Carol Graham und Andrés Solimano. Sie publizieren zunächst Aufsätze mit einer globalen Perspektive und führen Lateinamerika nur als eine unter vielen Regionen an. Das Interesse an den «Mittelklassen» ist verbunden mit der Idee, dass Gesellschaften, in denen mehr Einkommen in die mittleren Einkommenskategorien fliessen, gleichere Gesellschaften sind. Sie spitzen demnach die Ungleichheitsfrage auf die Frage nach der «Mittelklasse» zu. Aus diesen Überlegungen erwächst die Frage, welche politischen und wirtschaftlichen Effekte eine grosse «Mittelklasse» hat. So untersucht bspw. Easterly (2001) mittels eines Samples von über einhundert Ländern die positiven Wirkungen geringer Ungleichheit auf Entwicklungsprozesse. Diese bestehen in der Regel in höherem Wachstum, höherem Humankapital, besserer Gesundheit (z.B. niedrigere Kindersterblichkeitsraten und allgemein höhere Lebenserwartungen) und höheren Investitionen in die Infrastruktur. Solimano (2008) zeigt in einer ähnlich umfangreichen Untersuchung, dass es einen statistischen Zusammenhang zwischen ökonomischem Wohlstand der mittleren Bevölkerungsmehrheit und stabiler Demokratie, mehr Umverteilung und geringerer ökonomischer Ungleichheit gibt. Daraus schliesst er auf die wirtschaftliche und politische Relevanz einer grossen und stabilen «Mittelklasse» für Demokratisierungsprozesse, Wirtschaftswachstum und Ungleichheitsreduzierung.

Das zentrale Interesse der Entwicklungsökonomen wechselte in der Folge von den Effekten der Strukturanpassungsprogramme auf Armut und untere Einkommenskategorien auf die Wirkung der wirtschaftspolitischen Veränderungen auf die mittleren Einkommenskategorien (Birdsall et al. 2000). Die in dieser Zeit erschienenen Publikationen beschäftigen sich mit den Effekten der marktliberalen Strukturanpassungsprogramme auf die Berufs-, Einkommensund Bildungsstruktur und folgern daraus, was dies speziell für die «Mittelklassen» der Region bedeutet. Der Anteil formal sicherer und sozialversicherter Stellen mit mittleren und gehobenen Einkommen ging infolge der Strukturanpassungsprogramme zurück und wurde durch unsichere und nicht versicherte Stellen im nun stärker von internationalen Kapitalflüssen ab-

\footnotetext{
${ }^{15}$ Verschiedene Autor_innen weisen darauf hin, dass die zunehmende Auseinandersetzung der Ökonom_innen mit dem Thema Ungleichheit nicht nur inhaltliche Gründe hat, sondern auch eine Folge neuer Datengrundlagen und Analysemöglichkeiten ist (siehe bspw. Kanbur/Lustig 1999; Gasparini et al. 2009). Über lange Zeit fehlte die Datengrundlage für eine umfassende Analyse sozialer Ungleichheit in Lateinamerika. Erst mit der Etablierung von Datenbanken, basierend auf sukzessive eingeführten Household Surveys in fast allen lateinamerikanischen Ländern, wurde es möglich, soziale Ungleichheit und vor allem ihre Entwicklung systematisch zu untersuchen.
} 
hängigen privatwirtschaftlichen Bereich ersetzt (Rodrik 2001; Birdsall et al. 2000; Solimano 2006b). Sekundäre Schulabschlüsse, die zuvor zur Beschäftigung in der öffentlichen Verwaltung und in von der Importsubstitution geschützten Betrieben befähigt und einen relativ gehobenen Lebensstil ermöglicht hatten, büssten an Wert ein, da viele Stellen durch Verschlankung und Privatisierung des öffentlichen Sektors abgebaut und nicht im gleichen Umfang und gleichwertig mit Stellen im Privatsektor kompensiert worden waren (Birdsall et al. 2000; Graham 2002). Hinzu kam, dass das Wirtschaftswachstum weniger Arbeitsplätze geschaffen hatte als erwartet (,jobless-growth”, Birdsall 2007b: 7). Marktintegration, Liberalisierung und technologischer Wandel hatten zudem - anders als erhofft - nicht zu einer erhöhten Nachfrage nach unqualifizierter und gering qualifizierter Beschäftigung, sondern zu einer erhöhten Nachfrage nach Hochqualifizierten geführt (Behrman/Birdsall/Szekely 2001).

Während die Folgen der Strukturanpassungsprogramme auf die unteren Einkommensgruppen durch die Implementierung von auf Armut fokussierten Sozialprogrammen in den 1990er Jahren, den Ausbau des Pimarschulbereichs und die veränderte Arbeitsmarktstruktur abgefedert wurden, waren die «Mittelklassen» von Verarmung, Prekarisierung und drohendem Abstieg betroffen (Birdsall et al. 2000; Graham/Pettinato 2002; Solimano 2008). Die Abnahme gesicherter Stellen führte zu einem Anstieg der Arbeitslosigkeit innerhalb der «Mittelklassen» oder der Reintegration in oft wenig abgesicherte privatwirtschaftliche Stellen mit geringem Einkommen, um die sie nun mit vormals «Armen», die bislang kaum formaler Bildungsabschlüsse hatten, konkurrierten (Birdsall et al. 2000). Dies hatte zur Folge, dass die Arbeitslosigkeit von Personen mit Primarschulabschluss Ende der 1990er Jahre geringer war als die der Personen mit Sekundarschulabschluss (vgl. Graham/Sukhtankar (2002). Am oberen Ende der Einkommensverteilung war der wohlhabende Teil der Bevölkerung durch Besitz, Hochschulbildung, Möglichkeiten des Zugriffs auf Kredite und die Ausstattung ihrer Haushalte wesentlich weniger den zunehmenden Volatilitäten der internationalen Märkte ausgesetzt (Solimano 2006b). Zusätzlich profitierte die «Oberklasse» vom finanziellen Ausbau der Universitäten, die - nach wie vor - vor allem ihr vorbehalten waren. Dies habe, so Birdsall et al. (2000), zu einer zunehmenden Entfernung des obersten Einkommensdezils vom Rest der Bevölkerung geführt. Sie kommen daher zu dem Schluss, dass es zu Beginn des Jahrtausends den «Oberklassen» und «Armen» besser geht als in den 1980er Jahren, während das Bild für die «Mittelklassen» komplexer ist („middle-class squeeze”).

Es konnten in dieser Phase nur wenige Publikationen gefunden werden, die die relative Grösse der "Mittelklassen» und ihre sozioökonomischen Merkmale empirisch untersuchten (bspw. Birdsall et al. 2000; Solimano 2008; OECD 2010). In diesen Publikationen wird auf relative «Mittelklasse»-Definitionen aus der Diskussion um die Erosion der «Mittelklasse» in den Vereinigten Staaten zurückgegriffen (Thurow 1987; Davis/Huston 1992; Levy 1987). Die «Mittelklasse» liegt bei diesen Definitionen in einem definierten Bereich im Zentrum der Einkommensverteilung, sie wird also relativ zum Medianeinkommen bestimmt. Je nach Enge oder Breite dieses Bereichs um den Median ist die «Mittelklasse» unterschiedlich gross. Birdsall et al. (2000) rechnen bei einer verhältnismässig engen Definition von $75 \%$ bis $125 \%$ des Medianeinkommens $22 \%$ der lateinamerikanischen Bevölkerung der «Mittelklasse» zu. ${ }^{16}$ Mit

\footnotetext{
${ }^{16}$ Die Untersuchung bezieht sich nicht auf ganz Lateinamerika, sondern auf Brasilien, Chile, Costa Rica, Mexiko, Panama und Peru.
} 
den Schwellenwerten $50 \%$ bis $150 \%$ des Medians kommt die OECD einige Jahre später auf eine «Mittelklassen»-Grösse je nach Land zwischen etwa $34 \%$ und 53 \% (OECD 2010). Solimano (2008) bestimmt die «Mittelklasse» über die Einkommensperzentile drei bis neun. Sie macht demnach qua Definition 70 \% der Bevölkerung aus. Für das Jahr 2000 kommt er so zu einem «Mittelklassen»-Einkommensanteil für Lateinamerika von insgesamt 56,81 \%. ${ }^{17}$

Bei der Betrachtung von Einkommens-, Bildungs- und Gesundheitsindikatoren der «Mittelklasse» stellt Birdsall (2002) fest, dass ein grosser Teil der «Mittelklasse» sich kaum oder gar nicht von den von Armut betroffenen Bevölkerungsgruppen unterscheidet. Sie konstatiert ausserdem, dass hohe Preise und geringe Beschäftigungsraten in Krisenzeiten «Mittelklasse»Angehörige besonders hart treffen und diese ihren Lebensstandard kaum halten können. Solimano $(2008$; 2009) zeigt, dass die Einkommen der Mehrheit der Bevölkerung Lateinamerikas nicht weit streuen und an der Grenze zu extremer Armut liegen, sodass die lateinamerikanische «Mittelklasse» die ärmste der Welt ist. ${ }^{18}$ Durch die sehr schiefen Einkommensverteilungen bzw. die Ballung vieler Personen am unteren Ende der Einkommensverteilung leben $30 \%$ bis $40 \%$ der relativ definierten «Mittelklassen» Lateinamerikas unterhalb der nationalen absoluten Armutsschwellen (Cruces et al. 2011; Castellani et al. 2015). Solimano (2008) hält es daher nicht für zielführend, von einer «Mittelklasse» zu sprechen. Er unterscheidet stattdessen zwischen der «unteren» (drittes bis sechstes Perzentil) und der «oberen Mittelklasse» (siebentes bis neuntes Perzentil).

Die beobachtete sozio-ökonomische Prekarisierung und Destabilisierung der «Mittelklassen» bringt die meisten Autor_innen in dieser Phase zu einer relativ kritischen Bewertung der Strukturanpassungsprogramme. Zwar seien nicht wenige Personen aus der Armut in die untere «Mittelklasse» aufgestiegen, die Reformen des Washington Consensus trügen jedoch zu wenig dazu bei, die Stabilität in diesen Positionen zu gewährleisten. Die Publikationen konstatieren, dass die «Mittelklassen» durch die Reformprogramme zu wenig adressiert wurden, weil ihre wirtschaftliche und politische Rolle systematisch unterschätzt worden sei (bspw. Birdsall et al. 2000; Solimano 2005; Estache/Leipziger 2009a). Es sei gerade die «Mittelklasse», die sich für weitere markt- und globalisierungsfreundliche Reformen einsetze und die entscheidend für den Gesellschaftsvertrag sei. Die Erfahrung zeige, dass eine gesicherte «Mittelklasse» in den reifen Marktwirtschaften politisch für eine vernünftige Wirtschaftspolitik (Marktöffnung, Preisstabilität) stimme, während die «Armen» und «Vulnerablen» für wirtschaftspolitisch unvorteilhafte Optionen votierten (Birdsall 2007a). Eine beachtliche Anzahl an Autor_innen kommt zu dem Schluss, dass ein neues Paradigma notwendig ist, das auf die Mitte (verstanden als die Mehrheit) der Gesellschaft abzielt, damit die Globalisierung eine Verbesserung der Situation für alle bringt und diese nachhaltig ist. Dieses sehen sie in der wirtschafts-, finanzund sozialpolitischen Erweiterung des Programms des Washington Consensus hin zu einem Programm des Inclusive Growth, das neben den Zielen Wachstum und Armutsreduktion ver-

${ }^{17}$ In den Texten von Autor_innen, die mit Perzentildefinitionen arbeiten, wie Solimano und Easterly, verschwimmen häufig die Begriffe grösserer Einkommensanteil der «Mittelklasse» und grössere «Mittelklasse». Die Grösse der «Mittelklasse» ist aufgrund der Definition über Perzentile qua Definition stabil. Der Einkommensanteil dieser Gruppe ist demnach das eigentlich Interessante, er sollte aber nicht mit der «Mittelklassen»-Grösse verwechselt werden.

${ }^{18}$ Auf die drei mittleren Quintile der Bevölkerung (entspricht $60 \%$ ) fallen nur 56 \% des Gesamteinkommens. 
stärkt auf die Mitte der Gesellschaft zielt (vgl. Birdsall/La Torre 2001; Solimano 2005; 2006b; Molina 2006; Birdsall/La Torre/Menezes 2008; Huber 2009; Estache/Leipziger 2009a; Kremer/van Lieshout/Went 2009). ${ }^{19}$

Zusammenfassend lässt sich festhalten, dass die Beschäftigung mit der «Mittelklasse» in dieser ersten Phase aus der entwicklungsökonomischen Forschung und Diskussion zum Zusammenhang von Einkommensungleichheit und wirtschaftlichem Wachstum hervorgeht. Das zentrale Forschungsinteresse in dieser Phase gilt nicht der «Mittelklasse» an sich, sondern einer Wirtschafts- und Sozialpolitik, die diese Gruppe adressiert, um positive Makro-Effekte wie höheres Wirtschaftswachstum und politische Stabilität herbeizuführen. Es geht also um die Rolle der «Mittelklasse» für den wirtschaftlichen und politischen Wandel resp. Stabilität. Die ökonomische «Mittelklasse»-Forschung zu Lateinamerika startet in dieser Phase als Analyse und kritische Reflexion der sozialen Effekte der marktliberalen Strukturanpassungsprogramme auf die Mitte der Gesellschaft. Die Mehrzahl der Autor_innen kommen zu dem Schluss, dass die Lebensbedingungen der für Wirtschaft und Demokratie wichtigen «Mittelklassen» durch die Reformen prekarisiert worden sind und appellieren für eine Anpassung der Sozial- und Wirtschaftspolitik. Obwohl den lateinamerikanischen «Mittelklassen» positive Wertorientierung und Effekte auf Wirtschaft und Gesellschaft zugeschrieben werden, gibt es zu diesem Zeitpunkt kaum empirisch gesichertes Wissen über diese. Die Zuschreibungen basieren meist auf theoretischen Überlegungen, Ergebnissen der Ungleichheitsforschung und Ländervergleichen (bspw. Barro 1999a; Easterly 2001). Dies beklagen am Ende der Dekade auch zentrale Autor_innen wie zum Beispiel Andrés Solimano (2009).

\section{Phase II - Wirtschaftsaufschwung und die «aufsteigende Mittelklasse» (2010 bis 2018)}

Am Ende der ersten Dekade des Jahrtausends entstehen in der ökonomischen Ungleichheitsforschung erste Artikel, die rückblickend eine grundsätzliche Veränderung der wirtschaftlichen Entwicklung und von Ungleichheitsindikatoren für Lateinamerika seit etwa 2000 feststellen. ${ }^{20}$ Sie zeigen, dass das Wirtschaftswachstum seit der Jahrtausendwende gestiegen war, die inzwischen auch von den Ökonom_innen als problematisch erkannte Ungleichheit kontinuierlich sank, während die Armut sich stark reduziert habe (Gasparini/Cruces/Tornarolli/Marchionni 2009; Lustig/López-Calva 2010; Birdsall/Lustig/McLeod 2011). ${ }^{21}$ Die zweite, nicht weniger wichtige Feststellung ist, dass die Finanzkrise im Jahr 2009

${ }^{19}$ Die politischen Forderungen sind sehr unterschiedlich und werden nicht von allen Autor_innen geteilt. Beispiele sind: faires Steuer- und Umverteilungssystem mit einem höheren Steueraufkommen, das auch deficit spending ermöglicht; Minimaleinkommen; verbesserter Zugang zu Kapital und Krediten; universelle statt auf Armut zielende Sozialprogramme, sodass die «Mittelklasse» erreicht wird und sie ebenfalls von ihren Steuerzahlungen profitiert; mehr politische Partizipation.

${ }^{20}$ Zahlreiche Forschungsarbeiten zu Veränderungen der Ungleichheit in verschiedenen Ländern Lateinamerikas wurden beispielsweise durch das UNDP-Projekt „Markets, the State and the Dynamics of Inequality in Latin America” (LópezCalva/Lustig 2010) angestossen und finanziert.

${ }^{21}$ Obwohl schon Publikationen zwischen 2006 und 2010 eine sukzessive realwirtschaftliche Veränderung beobachten, fokussieren sie auf den Zeitraum davor (bspw. Solimano 2006a; Huber 2009). Neuere umfangreiche Sammelbände zur Entwicklung und $\mathrm{zu}$ den Ursachen sozialer Ungleichheit in Lateinamerika sind Cornia (2014a), Fritz (2015) und Bértola/Williamson (2017). Bemerkenswerterweise zweifelt Solimano (2016) auf der Grundlage mehrerer Länderstudien den Be- 
die Wirtschaften Lateinamerikas wesentlich weniger aus dem Gleichgewicht gebracht hat als die vieler westlicher Länder (OECD 2010).

Erstmals veröffentlichen grosse internationale Entwicklungsorganisationen umfassende Sammelbände zu «Mittelklassen» in Lateinamerika (OECD 2010; Ferreira et al. 2013; DaytonJohnson 2015a). ${ }^{22}$ Im Folgenden werden die Ergebnisse zu den wichtigste Forschungsfragen zusammengestellt, welche die meisten empirischen Arbeiten in dieser Phase adressieren.

\section{Wächst die «Mittelklasse»?}

Trotz der positiven wirtschaftlichen Entwicklung in der ersten Dekade konnte unabhängig von den verwendeten Definitionen zunächst keine oder nur eine marginale Grössenveränderung der «Mittelklasse» nachgewiesen werden. Vielmehr kamen verschiedene Publikationen am Ende der ersten Phase zu dem Ergebnis, dass im Durchschnitt keine nennenswerten Veränderungen der relativen Grösse der «Mittelklasse» zu beobachten sind (Hertova/LópezCalva/Ortiz-Juárez 2010; Cruces et al. 2011; Castellani/Parent 2011). Da sich die Schwellenwerte bei relativen «Mittelklasse»-Definitionen mit der Einkommensverteilung verschieben, bleiben allgemeine Einkommenssteigerungen in der Gesamtgesellschaft empirisch unsichtbar. Der Umfang der «Mittelklasse» ändert sich bei relativen Definitionen nur, wenn sich etwas in der Relation der Einkommen verändert. Die Relation der Einkommen war aber in dieser Phase recht stabil. Aus diesem Grund werden diese Definitionen von López-Calva und OrtizJuaréz (2011) verworfen und die Verwendung einer absoluten Definition mit einem unteren Schwellenwert von $\$ 10$ und einem oberen Schwellenwert von $\$ 50$ empfohlen. ${ }^{23}$ Der vergleichsweise hohe untere Schwellenwert wird damit begründet, dass neben einer minimalen Kaufkraft auch relative ökonomische Sicherheit für die «Mittelklasse» konstitutiv sei. Die Autor_innen stellen empirisch ${ }^{24}$ fest, dass die Wahrscheinlichkeit des Abstiegs in Armut ab der \$10-Grenze unter $10 \%$ sinkt (López-Calva/Ortiz-Juárez 2011).

Mit dieser Definition kann für einige lateinamerikanische Länder ein moderates bis starkes «Mittelklasse»-Wachstum in der ersten Dekade diagnostiziert werden. ${ }^{25}$ Für ganz Lateinamerika ergeben die neuen Berechnungen einen Anstieg des "Mittelklasse»-Anteils von etwa einem Fünftel auf etwa ein Drittel der Bevölkerung (vgl. Ferreira et al. 2013; Birdsall 2012). In

fund an, dass die Ungleichheit grundsätzlich sinke. Er zeigt, dass der GINI, berechnet auf der Grundlage von Steuerdaten, stabil ist und in einigen Ländern sogar steigt.

${ }^{22}$ Diese Publikationen werden begleitet von einer Vielzahl von Artikeln derselben Autor_innen, die die Ergebnisse fortschreiben und tiefere Einsichten in die Forschungsprozesse gewähren: OECD: Daude/Melguizo 2010; Castellani/Parent 2011; Daude 2011; 2013; Dayton-Johnson 2011. Weltbank/UNDP/IDB: Hertova et al. 2010; Cruces et al. 2011; López-Calva/OrtizJuárez 2011; Lora/Fajardo 2011; 2013; Rigolini 2012; Ferreira et al. 2013; López-Calva 2013; López-Calva/Cruces/Lach/OrtizJuárez 2014; UNDP 2014.

${ }^{23}$ Vor dem hier beschriebenen Zeitraum entfachte bereits eine Diskussion um die Entstehung einer globalen «Mittelklasse» und um «Mittelklassen» in periphären Weltregionen (Milanovic 2005; IBRD/The World Bank 2007; Wilson/Dragusanu 2008; Kharas 2010; Kharas/Gertz 2010) Dort wurden von Milanovic/Yitzhaki (2002), Banerjee/Duflo (2008) und Ravallion (2009) bereits absolute «Mittelklasse»-Definitionen eingeführt. Birdsall (2010) appliziert die Definition von Milano$\mathrm{vic/Yitzhaki} \mathrm{(2002)} \mathrm{leicht} \mathrm{verändert} \mathrm{auf} \mathrm{die} \mathrm{lateinamerikanischen} \mathrm{Verhältnisse.}$

${ }^{24}$ Die Untersuchung arbeitet mit Daten aus den drei Ländern Chile, Peru und Mexiko.

${ }^{25}$ Empirische Ergebnisse von Birdsall (2010): Land ([Anteil «Mittelklasse» 1990] - [Anteil «Mittelklasse» 2005]): Starkes Wachstum der «Mittelklasse»: Mexiko (18 \%-28 \%); Honduras (0 \%-7 \%); Paraguay (5 \%-18 \%); Chile (20 \%-33 \%); Moderates Wachstum der «Mittelklasse»: Bolivien (8\%-12\%), Kolumbien (10\%-14\%); Ecuador (10\%-14\%); Brasilien (16\%$19 \%)$. Schrumpfung der «Mittelklasse»: Venezuela (20\%-3\%); Argentinien (39\%-30\%). 
der Folge dieser Feststellung werden absolute Grenzwerte zum Standard in den ökonomischen Operationalisierungen von «Mittelklassen» (mit Ausnahme von OECD-Studien). Castellani/Parent (2015) gelingt der Nachweis des «Mittelklasse»-Wachstums auch für eine relative Definition (50\% bis $150 \%$ des Medianeinkommens).

Wie gross ist die «Mittelklasse»?

Wir halten es auf der Basis der gesichteten Publikationen nicht für möglich, eine klare Aussage über die Grösse und genaue Entwicklung der «Mittelklasse» in Lateinamerika zu treffen. Die folgende Auswahl von empirisch ermittelten «Mittelklasse»-Grössen macht dies deutlich: 30,5 \% (Stampini et al. 2016), 34,5 \% (CEPAL 2018), 48 \% (Tornarolli 2014) und 66 \% (Lora/Fajardo 2011). Die angegebenen Grössen hängen vom genauen Jahr, der Definition, der Datengrundlage, dem Berechnungsverfahren, dem Ländersample und der Grundgesamtheit ab. Dies führt dazu, dass Autor_innen selbst bei gleichen Definitionen zu erheblich anderen «Mittelklasse»-Grössen zum gleichen Zeitpunkt für dasselbe Land kommen. ${ }^{26}$ Leider werden die unterschiedlichen Ergebnisse von den Autor_innen übergangen, obwohl sie die jeweils anderen Publikationen zur Kenntnis nehmen. Auch die Frage, ob aufgrund der erheblichen nationalen Unterschiede Aussagen über ganz Lateinamerika überhaupt zulässig sind, wird nicht thematisiert.

\section{Welche Ursachen hat die «Expansion der Mittelklasse»?}

In nur wenigen Publikationen wird die Frage aufgeworfen, warum die «Mittelklassen» überhaupt wachsen. Sofern Ursachen genannt werden, werden diese nicht eigens empirisch untersucht, sondern es wird auf Ergebnisse der ökonomischen Ungleichheitsforschung rekurriert. Als wichtige Faktoren für das Wachstum der «Mittelklassen» werden die abnehmende Einkommensungleichheit in lateinamerikanischen Gesellschaften sowie das Wirtschaftswachstum in der ersten Dekade genannt. Das Wirtschaftswachstum wird als Erklärung für den Anstieg des Einkommensanteils der «Mittelklassen» am Gesamteinkommen angeführt. Allerdings betonen Ferreira et al. (2013), dass das «Mittelklasse»-Wachstum nicht ausschliesslich auf das Wirtschaftswachstum zurückgeführt werden kann, denn lateinamerikanische Länder mit vergleichbarem Wirtschaftswachstum verzeichneten sehr unterschiedliche Entwicklungen der «Mittelklasse». Entscheidend für das «Mittelklasse»-Wachstum in einem Land sei, dass sich das Wirtschaftswachstum in geringerer sozialer Ungleichheit und höheren Durchschnittslöhnen niederschlage (vgl. dazu auch Cárdenas et al. 2015). Ferreira et al. (2013) nennen das Sinken der im internationalen Vergleich zuvor hohen Bildungsdividende (vor allem für tertiäre Zertifikate) als wichtigen Faktor für den Rückgang der sozialen Ungleichheit. Für das Absinken der Bildungsdividende, d.h. das Ausmass, in dem mehr formale Bildung zu mehr Einkommen führt, werden wiederum zwei Hauptfaktoren angeführt. ${ }^{27}$ Einige Autor_innen führen den Ausbau des Bildungssystems und den daraus folgenden Anstieg der

${ }^{26}$ Selbst mit der gleichen «Mittelklasse»-Definition kommen unterschiedliche Publikationen für das gleiche Jahr auf erheblich unterschiedliche Grössen, bspw. Chile im Jahr 2011: 65,7 \% (Castellani/Parent 2015); 56,8 \% (Solimano 2016); 45,7 \% (Stampini/Robles/Sáenz//Ibarrarán/Medellín 2016). Die Berechnung wird häufig nicht genauer spezifiziert. Typische Unterschiede bestehen aber darin, ob Pro-Kopf-Einkommen verwendet werden oder eine Äquivalenzskala (die ebenfalls variieren kann), sowie darin, welches die zu kategorisierende kleinste Einheit ist (Haushalt oder Individuum).

${ }^{27}$ Für einen Überblick, siehe Lustig/López-Calva/Ortiz-Juárez/Monga (2016). 
Schul- und Universitätsabschlüsse als Ursache an (Daude 2011; 2012; Cruces et al. 2011; López-Calva/Lustig 2012). Dieses grössere Angebot an gebildeten und hochgebildeten Arbeitskräften führe zu einem Sinken der entsprechenden Bildungsdividende und damit zur Verringerung der Unterschiede der Markteinkommen (López-Calva/Lustig 2010; Gasparini/Cruces/Tornarolli 2011; Lustig et. al. 2016; Azevedo/Inchaust/Sanfelice 2013; Andersson/Palacio 2017) ${ }^{28}$. Andere Autor_innen nennen vordergründig die veränderte Nachfragestruktur nach Arbeitskräften. Durch den Exportboom und den starken Anstieg von Auslandsinvestitionen habe sich der Arbeitsmarkt für gering qualifizierte Arbeitskräfte deutlich verbessert (Cornia 2014b; Andersson/Palacio 2017; La Torre/Messina/Silva 2017; Azevedo/López-Calva/Lustig/Ortiz-Juárez 2015). Neben den veränderten Bildungsdividenden werden in der ökonomischen Ungleichheitsforschung auch die Conditional-Cash-TransferProgramme $(C C T)^{29}$ und staatlichen Eingriffe in den Arbeitsmarkt durch die Einführung von Mindestlöhnen thematisiert (vgl. bspw. López-Calva/Lustig 2012; Paes de Barros/Ferreira/Molinas Vega/Saavedra Chanduvi 2009; Lustig et al. 2016). Die Transfers seien für etwa $20 \%$ des «Mittelklasse»-Wachstums verantwortlich, die veränderte Primärverteilung der Markteinkommen für 80 \% (Ferreira et al. 2013).

Die ökonomische Forschung analysiert vor allem Makroindikatoren und fokussiert auf die Veränderung der Ungleichheit. Es fehlt an landesspezifischer empirischer Forschung bzw. an deren Rezeption, die etwa auf die veränderte Verhandlungsmacht zwischen Arbeitnehmer_innen und Arbeitgeber_innen oder die reduzierte Arbeitsplatzsicherheit fokussiert. Als einer von wenigen Ökonom_innen thematisiert Cornia (2014b) als wichtige Ursachen der abnehmenden Einkommensungleichheit den sozialen und politischen Widerstand gegen die negativen Auswirkungen der Globalisierung und die daraus resultierenden Veränderungen in der Arbeitsmarktpolitik (institutionalisierte Lohnverhandlungen, Formalisierung von Beschäftigung und Steuerpolitik). Die Auswirkungen der genannten Faktoren auf die Grösse der «Mittelklasse» und ihren Einkommensanteil werden jedoch auch hier lediglich angenommen und kaum empirisch belegt.

\section{Was charakterisiert die «Mittelklasse»?}

Anders als bei der ökonomischen Ungleichheitsforschung interessieren sich die Autor_innen der ökonomischen «Mittelklasse»-Forschung für die soziodemographischen Gemeinsamkeiten der Einkommenskategorie «Mittelklasse».

Einkommenssituation: Die über absolute Schwellenwerte definierten «Mittelklassen» liegen nicht automatisch in der Mitte der Einkommensverteilung, wie es die Bezeichnung suggeriert. Vielmehr gehören die so definierten «Mittelklassen» in Lateinamerika zu den oberen Einkommenskategorien. So zeigen bspw. Bussolo/Maliszewska/Murard (2017: 4), dass 2005 in Lateinamerika 80 \% der «Mittelklasse»-Angehörigen (definiert nach López-Calva/Ortiz-Juárez

\footnotetext{
${ }^{28}$ Das in Phase I vorgebrachte Argument von Birdsall et al. (2000) wird hier umgekehrt verwendet. In der ersten Phase sprachen die Autor_innen von einem Steigen der ohnehin schon hohen Bildungsdividende auf tertiäre Bildung.

${ }^{29}$ Die monetären Transfers (Conditional-Cash-Transfer-Programme) werden nur unter bestimmten Bedingungen ausgezahlt, wie der Schulbesuch von Kindern oder die Gesundheitsvorsorge. Die Wahrscheinlichkeit eines Aufstieges aus der Armut soll damit erhöht werden (vgl. Fiszbein/Schady/Ferreira/Grosh/Keleher/Olinto/Skoufias 2009; Saavedra/García 2012). Ferreira et al. (2013) beziffern zwar den Anstieg der Haushaltseinkommen durch die sozialen Transferprogramme, die Steuerungswirkung dieser Instrumente bleibt aber unthematisiert.
} 
2011) zum neunten und zehnten Einkommensdezil gehörten. Nach Birdsall (2012) liegt die in dieser Weise definierte «Mittelklasse» in den meisten lateinamerikanischen Ländern im obersten Quintil der Einkommensverteilung. Sie ist also vergleichsweise wohlhabend.

Familienform: Mit steigendem Einkommen sinkt die Familiengrösse. «Mittelklasse»Haushalte sind demnach im Vergleich zu armen Haushalten kleiner. Der typische «Mittelklasse»-Haushalt wird als Kernfamilie beschrieben, bestehend aus Eltern und Kindern (vgl. Birdsall 2012; Cruces et al. 2011; Castellani et al. 2015).

Bildung: Bildung und Einkommen korrelieren in allen Untersuchungen im lateinamerikanischen Kontext, d.h., je höher die Einkommensklasse ist, desto höher ist auch die Bildung. Tendenziell haben "Arme» eher Primarschulbildung, «Mittelklasse»-Angehörige eine Form von Sekundarschulbildung (abgeschlossen oder nicht) und «Oberklasse»-Angehörige eine abgeschlossene Sekundarschulbildung oder Universitätsabschlüsse (OECD 2010; Birdsall 2012; Ferreira et. al. 2013; Castellani/Parent 2011; Castellani et al. 2014). Unabhängig von der jeweiligen Definition können Cruces et al. (2011) zeigen, dass die Bildung in allen Einkommengruppen in den letzten Dekaden gestiegen ist.

Beschäftigungsverhältnisse: Häufig werden «Mittelklassen» in bestimmten Berufsfeldern oder Wirtschaftssektoren verortet, wie z.B. im öffentlichen Dienst, als (höhere) Angestellte in privaten Unternehmen und als Kleinunternehmer_innen. Empirisch ergibt sich aber für die mittleren Einkommenskategorien keine eindeutige Zuordnung zu Wirtschaftssektoren (Castellani/Parent 2011). Je nach Land gibt es Unterschiede, aber kein Wirtschaftssektor wird von einer Klasse dominiert (OECD 2010). Der Anteil der Angestellten im öffentlichen Dienst steigt stetig mit dem Einkommen. Daher arbeiten die höchsten Einkommenskategorien anteilig am häufigsten im öffentlichen Dienst (OECD 2010). Allerdings liegt der allgemeine Anteil an Stellen im öffentlichen Dienst in der Regel unter $20 \%$, sodass seine Bedeutung überschätzt würde (vgl. OECD 2010; Birdsall 2012).

Auch der Anteil formaler Beschäftigungsverhältnisse steigt wie das Bildungsniveau mit dem Einkommen (Cruces et al. 2011). Ob formale oder informelle Beschäftigungsverhältnisse in Armutslagen oder in der «Mittelklasse» dominieren, hängt daher von der «Mittelklasse»Definition ab. Informalität wird in jenen Publikationen als zentrales Merkmal der «Mittelklasse» genannt, die mit relativen Definitionen arbeiten (OECD 2010; Dayton-Johnson 2015a); hingegen ist in Publikationen, die absolute Definitionen verwenden, die Informalität eher ein Problem der «Vulnerablen» unterhalb der «Mittelklasse» (Ferreira et al. 2013; Rigolini 2012) und «Mittelklassen» selbst sind typischerweise formal angestellt oder selbstständig (Cruces et al. 2011).

Unternehmertum: Eine wesentliche Eigenschaft, die der «Mittelklasse» in der ökonomischen Literatur zugeschrieben wird, ist ihre Neigung zur Selbstständigkeit und zum Unternehmer_innentum. Aufgrund dieser ihr zugeschriebenen Eigenschaft wird sie als wichtig für die Innovationskraft und das Wachstum einer Wirtschaft betrachtet (Doepke/Zilibotti 2007; OECD 2010; Solimano 2014a; 2014b). Tatsächlich gehören in Lateinamerika die Mehrheit der Kleinunternehmer_innen oder Selbstständigen der «Mittelklasse» an. Allerdings arbeitet die Mehrzahl der Unternehmer_innen Lateinamerikas in Ein-Personen-Betrieben und in Bereichen, die weniger für grössere Innovationen stehen. Es ist daher davon auszugehen, dass es sich hier eher um Selbstständige aus Notwendigkeit und nicht um Selbstständige aus freier 
Wahl handelt (vgl. Banerjee/Duflo 2008; Solimano 2014a; 2014b; Castellani/Lora 2014; Mejía/Meléndez 2014). ${ }^{30}$ Erstere kommen eher aus der armen Bevölkerung oder aus der «Mittelklasse», letztere hauptsächlich aus der «Oberklasse» (vgl. Castellani/Lora 2014; OECD 2010; Birdsall 2012; Cruces et al. 2011).

\section{Welche Werte hat die «Mittelklasse»?}

Als wichtiger Begründungszusammenhang für die «Mittelklasse»-Forschung werden, wie bereits erwähnt, häufig Werte und Haltungen der «Mittelklasse» genannt, aus denen ihre besondere Rolle und Wichtigkeit für die Gesellschaft abgeleitet wird. Die Autor_innen beschreiben Angehörige der «Mittelklasse» als fleissig, leistungsorientiert, sparsam und an Stabilität interessiert. In Erweiterung zu diesen eher allgemeinen und positiven Zuschreibungen entstehen in Phase II Studien, die der Frage nach den politischen Werten der lateinamerikanischen «Mittelklassen» empirisch nachgehen. Typische Fragen sind, ob sich die lateinamerikanischen «Mittelklassen» grundsätzlich von den anderen Klassen durch ihre Einstellungen in Hinblick auf Demokratie, Toleranz, Bürgerrechte, Vertrauen in politische Institutionen unterscheiden und welche wirtschaftspolitischen Positionen bzgl. Marktöffnung, Steuergerechtigkeit, Umverteilung und Privatisierung sie haben (Daude/Gutiérrez/Melguizo 2015; Cárdenas et al. 2015; López-Calva/Rigolini/Torche 2012; Penfold/Rodríguez Guzmán 2014; Fierro 2015; OECD 2010; Gaviria 2007). Die Datengrundlage dieser Untersuchungen sind zumeist das Latinobarometro und das World Values Survey. Da in diesen Erhebungen das Einkommen nicht erhoben wird, wird die «Mittelklasse» unter Zuhilfenahme anderer Variablen, wie bspw. subjektive Selbsteinstufung oder Konsumausgaben, modelliert. Dabei wird angenommen, dass diese Variablen relativ gute Schätzer für das Einkommen sind (López-Calva et al. 2012; Fierro 2015). Die unterschiedlichen Datengrundlagen und «Mittelklassen»-Schätzer sind Gründe für das sehr uneinheitliche Bild der «Mittelklassen», welches durch diese Publikationen gezeichnet wird.

Das häufig angenommene Bekenntnis der «Mittelklassen» zum liberalen, demokratisch verfassten Staat, der für sozialen Ausgleich sorgt, wird nicht in dieser Klarheit gefunden. Die Zustimmungswerte zur Demokratie in Lateinamerika sind grundsätzlich, d.h. in allen Klassen, vergleichsweise gering (Fierro 2015). Welche Zustimmungswerte die «Mittelklassen» haben, ist nicht eindeutig und hängt letztlich von der jeweiligen Untersuchung ab. So zeigt beispielsweise die OECD (2010), dass die «Mittelklasse» die höchsten Zustimmungswerte zur Demokratie hat, wohingegen Fierro (2015) zeigt, dass die Zustimmungswerte stetig mit der Klasse steigen - wobei in beiden Fällen die prozentualen Unterschiede zwischen den Klassen gering sind. Wird auf detailliertere Variablen wie Toleranz oder Einstellung zu Bürgerrechten geschaut, wird das Bild noch unklarer. López-Calva et al. (2012) zeigen bspw., dass die «Mittelklasse» einerseits toleranter bezüglich anderer sozialer Gruppen und Identitäten ist und andererseits durchaus bereit ist, anderen sozialen Gruppen, wie „kriminellen“ Bürger_innen, die

\footnotetext{
30 „Die Mehrheit der Unternehmen von Armen und der Einkommensgruppe zwischen $\$ 2$ und \$10 weltweit sind kleine Geschäfte. Viele dieser Geschäfte haben den gleichen kleinen Warenbestand wie die anderen in der gleichen Strasse Grundnahrungsmittel, Süssigkeiten, Seife und ein paar weitere Produkte. Sie machen ein paar Verkäufe pro Tag und erzielen einen durchschnittlichen Gewinn von etwa 133 Dollar pro Jahr” (Kenny 2011, eigene Übersetzung). Allgemein zum Thema «Entrepreneure» in Lateinamerika: Kantis/Ishida/Komori (2002); Brenes/Haar (2014).
} 
Bürgerrechte abzuerkennen. Vertritt die «Mittelklasse» in Chile und Uruguay tendenziell linke politische Positionen, so ist sie bspw. in der Dominikanischen Republik eher rechts (Penfold/Rodríguez Guzmán 2014). Übereinstimmend wird in den Untersuchungen festgestellt, dass die «Mittelklassen» im Vergleich zu anderen Klassen moderate politische Positionen vertreten und eher nicht politisch aktiv sind (Penfold/Rodríguez Guzmán 2014).

Trotz der erheblichen Einkommensungleichheiten lässt sich kein eindeutiger Zuspruch für eine stärkere staatliche Umverteilung durch progressive Steuern identifizieren. So ist bspw. in Peru die «Mittelklasse» eher gegen Umverteilung, wohingegen sie in Uruguay eher dafür ist (vgl. Daude/Gutiérrez/Melguizo 2015). Die Bereitschaft Steuern zu zahlen, d.h. einen Beitrag zur Umverteilung und zur Finanzierung staatlicher Institutionen zu leisten, hängt mit dem Vertrauen in diese Institutionen und die Demokratie allgemein zusammen. In den Ländern, in denen die «Mittelklasse» den Institutionen weniger traut, ist sie auch weniger bereit, höhere Steuern in Kauf zu nehmen (Daude/Melguizo 2010; Fierro 2015). Im globalen Vergleich ist das Vertrauen der «Mittelklassen» Lateinamerikas in die staatlichen Institutionen relativ gering (Penfold/Rodríguez Guzmán 2014).

Die Untersuchungen stellen überraschenderweise fest, dass die empirisch festgestellten Werte der mittleren Einkommensklassen die Annahme typischer genuiner «Mittelklasse»Werte nicht stützen. Einige Werte steigen bzw. sinken stetig mit dem Einkommen und können daher nicht genuin als «Mittelklasse»-Werte interpretiert werden. Zudem variieren die Wertorientierungen der «Mittelklassen» z.T. stark nach dem jeweiligen Land. In Ländervergleichen zeigen sowohl Daude/Gutiérrez/Melguizo (2015) als auch López-Calva et al. (2012), dass die wenigen systematischen und signifikanten Unterschiede zwischen «Mittelklassen» und anderen Klassen niedriger sind als zwischen den verschiedenen Ländern Lateinamerikas.

Welche Wirkung hat die «Mittelklasse» auf gesellschaftliche Institutionen?

In der ersten Phase hatten statistische Analysen verschiedener Entwicklungsökonomen einen Zusammenhang zwischen einer grossen «Mittelklasse» und wirtschaftlichem Wachstum, stabileren Demokratien, höherem Humankapital und besserer Gesundheit in den Gesellschaften nachgewiesen. In der zweiten Phase gehen verschiedene Autor_innen nun empirisch der Frage nach, wie die «Mittelklassen» auf verschiedene gesellschaftliche Teilbereiche wirken. Dabei dominiert die zentrale Beobachtung in fast allen Untersuchungen, dass die «Mittelklassen» aus gesamtgesellschaftlichen Institutionen, bspw. Bildungseinrichtungen, Gesundheitsund Rentenversicherungssystemen, aussteigen, wenn sie keinen Vorteil für sich darin erkennen können (Banerjee/Duflo 2008; Ferreira et al. 2013; Daude/Gutiérrez/Melguizo 2015). Die Schulgängerquoten konnten durch die Conditional-Cash-Transfer-Programme im Untersuchungszeitraum zwar erhöht worden, die Qualität des Bildungssystems verschlechterte sich jedoch infolge einer enormen und rapiden Zunahme der Schülerzahlen in den meisten Ländern merklich. In Kombination mit dem Sinken der Bildungsdividende und die gleichzeitige Expansion privater Bildungsinstitutionen führte dies zu einem Austritt der oberen «Mittelklassen» aus den öffentlichen Bildungssystemen: Sobald sich Haushalte Privatschulen leisten können, tun sie dies in der Regel auch (OECD 2010; Birdsall 2012; Kremer et al. 2009; Daude 2012; Neri 2015; Larrañaga/Rodríguez 2014; Ferreira et al. 2013; Penfold/Rodríguez Guzmán 2014). Diese Beobachtung betrifft auch das Gesundheitssystem (Huber 2009; Neri 2015), das 
Rentensystem (Daude/de Laiglesia/Melguizo 2015) und das Steuersystem (vgl. Daude/Melguizo 2010; Cârdenas et al. 2015; Daude/Gutiérrez/Melguizo 2015). Ob die «Mittelklassen» den hohen Erwartungen an ihre gesellschaftliche Wirkung gerecht wird, hängt daher stark vom politischen Kontext und den lokalen und nationalen Interessen der «Mittelklassen» ab (vgl. Gilbert 2016). Es lassen sich demnach keine allgemeinen Annahmen diesbezüglich aus der schlichten Existenz einer «Mittelklasse» ziehen.

Der Literaturüberblick hat gezeigt, dass eine markante inhaltliche Veränderung im ökonomischen Forschungsstrang darin besteht, dass nicht mehr vorrangig eine zu kleine oder unsichere «Mittelklasse» beklagt wird, sondern endlich die lang erwartete lateinamerikanische «Mittelklasse» verkündet wird (Rigolini 2012; Dayton-Johnson 2015b; Birdsall 2016; Bussolo et al. 2017; Neri 2015). Durch den Wechsel von relativen zu absoluten Operationalisierungen der «Mittelklasse» wird sie weit weg vom Medianeinkommen in die oberen zwei oder drei Dezile der Einkommensverteilung verortet, die im nationalen Vergleich wohlhabend sind (Birdsall 2012). Darunter wird die neue Kategorie der «Vulnerablen» mit einem Pro-Kopf-Einkommen zwischen $\$ 4$ und \$10 eingeführt. Diese Einkommensgruppe lag in den relativen Operationalisierungen der Phase I noch im Zentrum der «Mittelklasse» und damit auch im Zentrum der Aufmerksamkeit. Auf der Prekarität und Instabilität der Lebenslage eben dieser Einkommensgruppe basierte die zentrale Kritik der Autor_innen an den Strukturanpassungsprogrammen. Das Thema und die Sorge um die «Vulnerabilität» wird durch diese definitorische Veränderung von der Kategorie «Mittelklassen» abgekoppelt, deren Aufstieg nun akklamiert werden kann. Dabei gerät aus dem Blick, dass die Kategorie der «Vulnerablen» in Lateinamerika die mit Abstand grösste Kategorie ist. «Vulnerable» und «Arme» zusammen bilden noch immer zwei Drittel der Bevölkerung (Ferreira et al. 2013). Auf der anderen Seite wird die Entwicklung der «Oberklasse» nicht mehr thematisiert. Sprechen Birdsall et al. (2000) in der ersten Phase noch von Top-Driven-Inequality, so taucht die «Oberklasse» in den Analysen nach 2010 kaum noch auf.

\section{Eine klassentheoretische Kritik ökonomischer «Mittelklassen»-Konzepte}

In den drei vorhergehenden Kapiteln wurde die ökonomische Diskussion um die lateinamerikanischen «Mittelklassen» systematisch dargestellt. Dabei wurde versucht, den Diskussionsverlauf und die divergierenden Ergebnisse der ökonomischen «Mittelklasse»-Forschung vor dem Hintergrund der unterschiedlichen Operationalisierungen der Mitte nachzuzeichnen. Trotz der z.T. grossen konzeptionellen Unterschiede liefern die gesichteten Publikationen interessante Hinweise zu relevanten gesellschaftlichen und wirtschaftlichen Veränderungsprozessen, die sich grob in zwei grundlegende Entwicklungstendenzen unterteilen lassen: die Prekarisierung, relative Verschlechterung oder Verarmung von Menschen aus mittleren sozialen Lagen infolge der Krisen und der Strukturanpassungsprogramme sowie die einkommens- und konsumbezogene Verbesserung unterer Einkommensgruppen seit Mitte der 2000er Jahre infolge der Zunahme des Pro-Kopf-Einkommens, der expandierten Kreditvergabe und der Bildungsexpansion. Diese Erkenntnisse beziehen sich auf die wirtschaftliche Ent- 
wicklung und die Veränderung der Einkommenssituation der lateinamerikanischen Bevölkerung und deren wirtschaftspolitische Ursachen.

Die Betrachtung von Einkommensverteilungen in Gesellschaften und ihre Veränderungen liefern wichtige Erkenntnisse über die Verteilung einer in heutigen Gesellschaften wichtigen Ressource, von der wiederum die Verfügung vieler anderer wichtiger Ressourcen abhängt. Die Unterteilung der Einkommensverteilung in Einkommensgruppen zur Eingrenzung sozialer Klassen halten wir jedoch aus soziologischer wie analytischer Sicht für problematisch. Je nach Definition - relativ oder absolut - verweisen Einkommensklassen-Konzepte auf «Konsumniveaus», «relativen Wohlstand» und «ökonomische Sicherheit». Wie auch bei soziologischen Berufsklassenmodellen sind die so gebildeten Klassen Makro-Aggregate, deren Klassenlage durch ökonomische Faktoren bestimmt wird. Die jeweiligen Aggregate können auf Grösse und Merkmale untersucht und mit sozioökonomischen Indikatoren wie das Wirtschaftswachstum ins Verhältnis gesetzt werden. Oberflächlich betrachtet handelt es sich in beiden Fällen um ähnliche Vorgehensweisen. Allerdings enthalten soziologische Klassenkonzepte wesentliche theoretische Implikationen, denen die von den Ökonom_innen verwendeten Einkommensaggregate (und ihre Proxys) nicht gerecht werden:

(i) Klassen sind relational: Klassenanalysen sind, trotz ihrer zahlreichen konzeptionellen Unterschiede, genuin relational angelegt, d.h. die sozialen Positionen von Akteuren oder sozialen Gruppen können immer nur in Relation zu anderen Akteuren oder sozialen Gruppen verstanden werden (Cosse 2014; Burzan 2011). Die Auswahl der Variablen bzw. Dimensionen für ein Klassenmodell wird in allen gängigen soziologischen Theorien theoretisch begründet. So wird bspw. von dem amerikanischen Soziologen Wright die Klassenlage wie bei Marx über das Ausbeutungsverhältnis bestimmt (Wright 1985). Ausbeutung selbst wiederum wird konzeptionalisiert über die verschiedenen Ressourcen Produktionsmittel, Organisationsmacht und Bildung. Diese werden dann operationalisiert und damit quantitativ messbar gemacht. So entsteht ein mehrdimensionales Feld von Klassenrelationen, in dem jede Klasse in einem spezifischen Verhältnis zu den anderen Klassen steht. Eine ähnliche Vorgehensweise gilt für das EGP-Schema (Erikson/Goldthorpe/Portocarero 1979) oder aktuelle Berufsklassenmodelle wie das Oesch-Modell (Oesch 2006), auch wenn in diesen Fällen das Verhältnis zwischen den Klassen anders bestimmt wird. Klassen sind vor diesem Hintergrund immer relationale Konstrukte.

(ii) Klassen sind Beziehungsgeflechte: Klassen sind „nicht bloss wechselnde und unbedeutende Aggregate, sondern Beziehungen, die sich im Laufe der Zeit zwischen Individuen und Familien und bestimmten Klassenpositionen herausbilden" (Goldthorpe 1985: 183). Die ökonomischen «Mittelklasse»-Ansätze vernachlässigen systematisch die Bedeutung von sozialen Strukturen wie Beschäftigungsstrukturen, Herrschaftsverhältnissen, sozialen Beziehungen oder Habitusmustern. So wird bspw. die Bedeutung der Beschäftigungsstruktur und damit der Position innerhalb der gesellschaftlichen Arbeitsteilung als Ursache ökonomischer Ungleichheit unzureichend thematisiert (Atkinson/Brandolini 2011). In Ermangelung einer hinreichenden Theoretisierung von Berufsstrukturen setzt sogar der sonst eher kritische Autor Solimano die «untere Mittelklasse» mit der Arbeiterklasse gleich, ohne dass er die entsprechenden Berufe 
und Lebensbedingungen betrachtet (Solimano 2016). Klassen sind keine Aggregate, denen Akteure aufgrund der gleichen sozialen Lage (Beruf, Einkommen, Bildung etc.) zugeordnet werden können (vgl. Graciarena 1967), sondern historisch gewordene und wandelbare Konfigurationen sozialer Beziehungen. In der marxistischen und neo-marxistischen Fassung des Begriffs wäre dies das Verhältnis zu den Produktionsmitteln. An Pierre Bourdieu und Edward P. Thompson anlehnende kultursoziologische Klassenkonzepte konzeptualisieren soziale Klassen als Gruppen mit geteilten Erfahrungen, ähnlichen Habitus und ähnlichen Ressourcen, die durch gemeinsame relative Positionen in gesellschaftlich relevanten Feldern (der Macht, der Arbeitsteilung etc.) entstehen (vgl. Bourdieu 1998; Thompson 1963; Vester/Oertzen/Geiling/Hermann/Müller 2001).

Die «Mittelklasse» als soziale Gruppe, ihre Lebensbedingungen und Haltungen bleiben aufgrund mangelnder Theoretisierung und Konzeptualisierung von «Klasse» und arbiträrer Definitionen der Mitte auffällig unterbeleuchtet, obwohl der «Mittelklasse»-Begriff im Zentrum der Publikationen steht. Für soziodemografische Merkmale, bei denen ein statistischer Zusammenhang zum Einkommen vorliegt (bspw. Bildungszertifikate, Anteil der Beschäftigten im öffentlichen Dienst, Familiengrösse), d.h. bei denen die Ausprägungen der Merkmale systematisch mit der Einkommenshöhe zusammenhängen, gilt, dass die «Mittelklasse» je nach Wahl der Schwellenwerte unterschiedliche Merkmale hat. Die Konstatierung typischer «Mittelklasse»-Merkmale ist somit zwangsläufig arbiträr. Publikationen zu politischen Einstellungen der «Mittelklasse» liefern eher unklare Zusammenhänge. Torche und López-Calva (2012) stellen daher selbstkritisch in Frage, ob Einkommen tatsächlich eine wesentliche Bestimmungsgrösse für diese Phänomene ist.

Aus soziologischer Sicht muss konstatiert werden, dass die ökonomische «Mittelklassen»Forschung genau genommen weder «Klassen» noch «Mittelklassen» beforscht und die in ihnen verwendeten «Mittelklassen»-Konzepte keine über die Einkommensverteilung hinausreichende Erklärungskraft haben. Zwar adressieren die Publikationen Fragen, die sich auf «Mittelklassen» als soziale Gruppe beziehen (Werte, Haltungen, soziodemographische Merkmale), ihre Konzeptualisierungen von «Mittelklasse» erfassen jedoch vor allem Veränderungen der Einkommensverteilung, allgemeines Einkommenswachstum sowie Einkommensmobilität - wie López-Calva und Ortiz-Juárez (2011) selbst als besonderen Vorteil der von ihnen vorgeschlagenen «Mittelklasse»-Definition betonen. Dieses Argument scheint aus unserer Sicht eher ein Argument gegen die Verwendung der Kategorie «Mittelklasse» zu sein. Denn wenn es lediglich darum geht, einkommensbasierte Trends abzubilden, so wäre die Beschreibung dieser Trends über die Einkommensvariable selbst wesentlich präziser. Dies wirft aus unserer Sicht die Frage auf, ob das Rekurrieren auf die «Mittelklasse» nicht vielmehr politische als wissenschaftliche bzw. analytische Gründe hat. Der «Mittelklasse»-Begriff ist in den diskutierten ökonomischen Publikationen positiv konnotiert. Mit ihm werden Wohlstand, Zufriedenheit, Stabilität, Demokratie und Lebensqualität in Verbindung gebracht. Die von den Autor_innen geforderten politischen Reformen lassen sich dadurch klarer formulieren und adressieren. Damit bietet der «Mittelklasse»-Begriff im Vergleich zu einer reinen Einkommensvariable den strategischen Vorteil, Fragen der Einkommensungleichheit nicht abs- 
trakt, sondern über eine soziale Kategorie zu thematisieren, die ein Identifikationspotential hat.

Vor dem Hintergrund der angeführten Kritik muss der analytische Wert der ökonomischen «Mittelklasse»-Konzepte für das Verständnis lateinamerikanischer Gesellschaften sowie der generelle Versuch, allgemeingültige Klassengrenzen und Klassenmerkmale zu finden und diese in international vergleichenden Studien auf unterschiedliche Gesellschaften anzuwenden, ${ }^{31}$ in Frage gestellt werden (vgl. auch Rehbein/Souza 2014). Wenn «Klassen» als soziale, historisch entstandene und veränderbare Gruppen verstanden werden, kann sich der Beantwortung der Frage nach ihrer Existenz, Anzahl, Grenzen und Merkmale nur empirisch durch gesellschaftsspezifische soziologische und historische Analysen von Klassenformationen und ihren symbolischen und materiellen (Re-)Produktionsmechanismen genähert werden (Wacquant 1991; Crompton 1993; Caínzos 2010; Parker 2012; Gayo 2013; Cosse 2014). ${ }^{32}$ Zudem wird durch die isolierte Untersuchung der mittleren sozialen Lagen die Relationen zwischen Klassen und damit Macht- und Ausbeutungsverhältnisse oder Konflikte ausgeblendet (Souza 2010). Dadurch gerät die relative Kontinuität sowohl der herrschenden Klasse als auch die der extremen Armut aus dem Blick.

\section{Literaturverzeichnis}

Adamovsky, Ezequiel Agustin (2013). "Clase media": reflexiones sobre los (malos) usos académicos de una categoría. Nueva Sociedad, 247, 38-49.

Aghion, Philippe, Eve Caroli und Cecilia García-Peñalosa (1999). Inequality and Economic Growth: The Perspective of the New Growth Theories. Journal of Economic Literature, 37, 1615-1660.

Alesina, Alberto und Dani Rodrik (1994). Distributive Politics and Economic Growth. The Quarterly Journal of Economics, 109(2), 465-490.

Altimir, Oscar (1996). Economic Development and Social Equity: A Latin American Perspective. Journal of Interamerican Studies and World Affairs, 38(2-3), 47-71.

Andersson, Martin und Andrés Palacio (2017). Inequality in Latin America: Agricultural Development, Inter-sectoral Duality, and the Kuznets Curve. In: Luis Bértola und Jeffrey G. Williamson (Hg.). Has Latin American Inequality Changed Direction? Looking Over the Long Run. Cham: Springer, 365-385.

Atkinson, Anthony Barnes (1997). Bringing Income Distribution in From the Cold. The Economic Journal, 107(441), 297-321.

Atkinson, Anthony Barnes und Andrea Brandolini (2011). On the Identification of the "Middle Class". Working Paper Nr. 217. ECINEQ, Society for the Study of Economic Inequality, Rom.

\footnotetext{
31 „En realidad, es un síntoma de la deriva de una sociología fuertemente teórica, cuantitativa, omniabarcante, con aspiraciones comparativas y con ello con una clara tendencia a la ubicuidad. El problema mencionado sirvió para denunciar una forma de hacer sociología, que parecía cada vez más distante de la sociedad que decía representar, lo cual implicaba un creciente desconocimiento de las distinciones que en la dinámica de la misma los individuos generan.” (Gayo 2013: 11)

${ }^{32}$ In der historischen und anthropologischen «Mittelklasse»-Forschung in Lateinamerika hat es vor einigen Jahren bereits diese Wende gegeben: Anstatt ihre Existenz a priori vorauszusetzen, widmen sich die Autor_innen der Frage, unter welchen soziopolitischen Bedingungen sich soziale Gruppen zu einer «Mittelklasse» formieren (vgl. Adamovsky 2013).
} 
Atria, Raúl (2004). Estructura ocupacional, estructura social y clases sociales. Serie Políticas Sociales Nr. 96. CEPAL, Comisión Económica para América Latina y el Caribe, Santiago de Chile.

Azevedo, João Pedro, Gabriela Inchaust und Viviane Sanfelice (2013). Decomposing the Recent Inequality Decline in Latin America. Policy Research Working Paper Nr. 6715. The World Bank, Washington, D.C.

Azevedo, João Pedro, Luis Felipe López-Calva, Nora Lustig und Eduardo Ortiz-Juárez (2015). Inequality, Mobility and Middle Classes in Latin America. In: Jeff Dayton-Johnson (Hg.). Latin America's Emerging Middle Classes. Economic Perspectives. London: Palgrave Macmillan UK, 32-50.

Banerjee, Abhijit Vinayak und Esther Duflo (2008). What is Middle Class about the Middle Classes around the World? The Journal of Economic Perspectives, 22(2), 3-28.

Bárcena Ibarra, Alicia und Narcís Serra (Hg.) (2010). Clases medias y desarrollo en América Latina. Chile: CEPAL; Fundación CIDOB.

Barro, Robert Joseph (1999a). Determinants of Democracy. Journal of Political Economy, 107(6), 158183.

Barro, Robert Joseph (1999b). Determinants of Economic Growth. A Cross-Country Empirical Study. Cambridge, Massachusetts: MIT Press.

Basu, Kaushik und Joseph Eugene Stiglitz (Hg.) (2016). Inequality and Growth. Volume II: Regions and Regularities. London: Palgrave Macmillan UK.

Behrman, Jere, Nancy Birdsall und Miguel Szekely (2001). Economic Policy and Wage Differentials in Latin America. PIER Working Paper Nr. 01-048. PIER, Penn Institute for Economic Research. University of Pennsylvania, Philadelphia, Pa.

Behrman, Jere Richard, Alejandro Gaviria und Miguel Székely (2001). Intergenerational Mobility in Latin America. Research Department Working Paper Series Nr. 452. IDB, Inter-American Development Bank, Washington, D.C.

Bhalla, Surjit (2007). Second Among Equals: The Middle Class Kingdoms of India and China [Draft, under revision]. ssbhalla.files.wordpress.com. Zugriff am 24.05.2019. ssbhalla.files.wordpress.com/2015/08/second_among_equals_-_omega_august-25-2015.pdf.

Birdsall, Nancy (2001). Why Inequality Matters. Some Economic Issues. Ethics \& International Affairs, 15(02), 3-28.

Birdsall, Nancy (2002). From Social Policy to an Open-Economy Social Contract in Latin America. Working Paper Nr. 21. CGD, Center for Global Development, Washington, D.C.

Birdsall, Nancy (2007a). Income Distribution: Effects on Growth and Development. Working Paper Nr. 118. CGD, Center for Global Development, Washington, D.C.

Birdsall, Nancy (2007b). Reflections on the Macro Foundations of the Middle Class in the Developing World. Working Paper Nr. 130. CGD, Center for Global Development, Washington, D.C.

Birdsall, Nancy (2010). The (Indispensable) Middle Class in Developing Countries, or: The Rich and the Rest, Not the Poor and the Rest. Working Paper Nr. 207. CGD, Center for Global Development, Washington, D.C.

Birdsall, Nancy (2012). A Note on the Middle Class in Latin America. Working Paper Nr. 303. CGD, Center for Global Development, Washington, D.C. 
Birdsall, Nancy (2016). Middle-Class Heroes: The Best Guarantee of Good Governance. Foreign Affairs, 2016 (march/april), 25-32.

Birdsall, Nancy, Carol Graham und Stefano Pettinato (2000). Stuck in the Tunnel: Is Globalization Muddling the Middle Class? Working Paper Nr. 14, Washington, D.C.

Birdsall, Nancy und Augusto de La Torre (2001). Washington Contentious: Economic Policies for Social Equity in Latin America. Washington, D.C.: Carnegie Endowment for International Peace; InterAmerican Dialogue.

Birdsall, Nancy, Augusto de La Torre und Rachel Menezes (2008). Fair Growth: Economic Policies for Latin America's Poor and Middle-Income Majority. Washington, D.C.: Center for Global Development (CGD); Inter-American Dialogue.

Birdsall, Nancy, Nora Lustig und Darryl McLeod (2011). Declining Inequality in Latin America: Some Economics, Some Politics. Working Paper Nr. 251. CGD, Center for Global Development, Washington, D.C.

Birdsall, Nancy, Nora Lustig und Christian Johannes Meyer (2014). The Strugglers: The New Poor in Latin America? World Development, 60, 132-146.

Boris, Dieter (2008). Sozialstrukturen in Lateinamerika. In: Dieter Boris, Therese Gerstenlauer, Alke Jenss, Kristy Schank und Johannes Schulten (Hg.). Sozialstrukturen in Lateinamerika. Ein Überblick. Wiesbaden: VS Verlag, 9-43.

Bourdieu, Pierre (1998). Praktische Vernunft. Zur Theorie des Handelns. Frankfurt am Main: Suhrkamp.

Burzan, Nicole (2011). Soziale Ungleichheit. Wiesbaden: VS Verlag.

Bussolo, Maurizio, Maryla Maliszewska und Elie Murard (2017). The Long-Awaited Rise of the Middle Class in Latin America Is Finally Happening. IZA Discussion Papers Nr. 10804. IZA, Institute of Labor Economics, Bonn.

Caínzos, Miguel (2010). Concepto y medida en el estudio de las clases medias en América Latina. Comentarios a la ponencia de Martín Hopenhayn. In: Ludolfo Paramio (Hg.). Clases medias y gobernabilidad en América Latina. Madrid: Fundación Pablo Iglesias, 59-68.

Cârdenas, Mauricio, Homi Kharas und Camila Henao (2015). Latin America’s Global Middle Class. A Preference for Growth over Equality. In: Jeff Dayton-Johnson (Hg.). Latin America's Emerging Middle Classes. Economic Perspectives. London: Palgrave Macmillan UK, 51-69.

Castellani, Francesca und Gwenn Parent (2011). Being "Middle-Class" in Latin America. Working Papers Nr. 305. OECD Development Centre, Paris.

Castellani, Francesca, Gwenn Parent und Jannet Zentero (2014). The Latin American Middle Class: Fragile After All? IDB Working Paper Series Nr. 557. IDB, Inter-American Development Bank, Washington, D.C.

Castellani, Francesca, Gwenn Parent und Jannet Zenteno Gonzales (2015). Who Is the Latin American Middle Class? Relative-Income and Multidimensional Approaches. In: Jeff Dayton-Johnson (Hg.). Latin America's Emerging Middle Classes. Economic Perspectives. London: Palgrave Macmillan UK, 101-129.

CEPAL, Comisión Económica para América Latina y el Caribe (2019). Panorama Social de América Latina 2018, Santiago. 
Cornia, Giovanni Andrea (Hg.) (2014a). Falling Inequality in Latin America. Policy Changes and Lessons. Oxford: Oxford University Press.

Cornia, Giovanni Andrea (2014b). Recent Distributive Changes in Latin America: An Overview. In: Giovanni Andrea Cornia (Hg.). Falling Inequality in Latin America. Policy Changes and Lessons. Oxford: Oxford University Press, 3-22.

Cosse, Isabella (2014). Las clases medias en la historia reciente latinoamericana. Historia y problemas del siglo XX, 5(5), 13-20.

Crompton, Rosemary (1993). Class and Stratification. An Introduction to Current De-bates. Cambridge: Polity Press.

Cruces, Guillermo, Luis Felipe López-Calva und Diego Battistón (2011). Down and Out or Up and In? Polarization-Based Measures of the Middle Class for Latin America. CEDLAS - Documento de Trabajo Nr. 113. CEDLAS, Centro de Estudios Distributivos, Laborales y Sociales, La Plata.

Daude, Christian (2011). Ascendance by Descendants? Working Paper Nr. 297. OECD Development Centre, Paris.

Daude, Christian (2012). Educación, clases medias y movilidad social en América Latina. In: Rebeca Grynspan und Ludolfo Paramio (Hg.). Clases medias en sociedades desiguales. Pensamiento Iberoamericano Nr. 10 (2012/1), AECID, Agencia Española de Cooperación Internacional para el Desarrollo, Madrid, 29-48.

Daude, Christian (2013). Education and Social Mobility in Latin America. LASA FORUM, XLIV(2), 7 9.

Daude, Christian, Hamlet Gutiérrez und Ángel Melguizo (2015). Political Attitudes of the Middle Class. The Case of Fiscal Policy. In: Jeff Dayton-Johnson (Hg.). Latin America's Emerging Middle Classes. Economic Perspectives. London: Palgrave Macmillan UK, 186-203.

Daude, Christian, Juan Ramón de Laiglesia und Ángel Melguizo (2015). Covering the Uncovered: Labor Informality, Pensions and the Emerging Middle Class in Latin America. In: Jeff DaytonJohnson (Hg.). Latin America's Emerging Middle Classes. Economic Perspectives. London: Palgrave Macmillan UK, 130-149.

Daude, Christian und Ángel Melguizo (2010). Taxation and more Representation? On Fiscal Policy, Social Mobility and Democracy in Latin America. Working Papers Nr. 294. OECD Development Centre, Paris.

Davis, Joe C. und John H. Huston (1992). The Shrinking Middle-Income Class. A Multivariate Analysis. Eastern Economic Journal, 18(3), 277-285.

Dayton-Johnson, Jeff (2011). Latinoamérica: la creación de una clase media. Política Exterior, 25(141), 156-170.

Dayton-Johnson, Jeff (Hg.) (2015a). Latin America's Emerging Middle Classes. Economic Perspectives. London: Palgrave Macmillan UK.

Dayton-Johnson, Jeff (2015b). Making Sense of Latin America's Middle Classes. In: Jeff DaytonJohnson (Hg.). Latin America's Emerging Middle Classes. Economic Perspectives. London: Palgrave Macmillan UK, 1-31.

Deininger, Klaus und Lyn Squire (1998). New Ways of Looking at Old Issues: Inequality and Growth. Journal of Development Economics, 57(2), 259-287. 
Doepke, Matthias und Fabrizio Zilibotti (2005). Social Class and the Spirit of Capitalism. Journal of the European Economic Association, 3(2-3), 516-524.

Doepke, Matthias und Fabrizio Zilibotti (2007). Occupational Choice and the Spirit of Capitalism. IZA Discussion Paper Nr. 2949. IZA, Institute of Labor Economics, Bonn.

Easterlin, Richard (1974). Does Economic Growth Improve the Human Lot? In: Paul David und Melvin Reder (Hg.). Nations and Households in Economic Growth. Essays in Honor of Moses Abramovitz. New York: Academic Press, 89-125.

Easterly, William (2001). The Middle Class Consensus and Economic Development. Journal of Economic Growth, 6(4), 317-335.

Erikson, Robert, John Harry Goldthorpe und Lucienne Portocarero (1979). Intergenerational Class Mobility in Three Western European Societies: England, France and Sweden. British Journal of Sociology, 30(4), 415-441.

Espinoza, Vicente (2002). La movilidad ocupacional en el Cono Sur. Acerca de las raíces estructurales de la desigualdad social. Revista Proposiciones, 34, 1-9.

Estache, Antonio und Danny Leipziger (Hg.) (2009a). Stuck in the Middle. Is Fiscal Policy Failing the Middle Class? Washington, D.C.: Brookings Institution Press.

Estache, Antonio und Danny Leipziger (2009b). Overview: Fiscal Policy, Distribution, and the Middle Class. In: Antonio Estache und Danny Leipziger (Hg.). Stuck in the Middle. Is Fiscal Policy Failing the Middle Class? Washington, D.C.: Brookings Institution Press, 1-23.

Ferranti, David de, Guillermo Perry, Francisco H. G. Ferreira und Michael Walton (2004). Inequality in Latin America: Breaking with History? Washington, D.C.: The World Bank.

Ferreira, Francisco H. G., Julian Messina, Jamele Rigolini, Luis Felipe López-Calva und Maria Ana Lugo (2013). Economic Mobility and the Rise of the Latin American Middle Class. Washington, D.C.: The World Bank.

Fierro, Jaime (2015). Clase Media y democracia en América Latina. Perfiles Latinoamericanos, 23(46), $37-60$.

Filgueira, Carlos (2001). La actualidad de viejas temáticas. Sobre los estudios de clase, estratificación y movilidad social en America Latina. Serie Políticas Sociales Nr. 51. CEPAL, Comisión Económica para América Latina y el Caribe, Santiago de Chile.

Fiszbein, Ariel, Norbert Schady, Francisco Ferreira, Margaret Grosh, Niall Keleher, Pedro Olinto und Emmanuel Skoufias (2009). Conditional Cash Transfers. Reducing Present and Future Poverty. The World Bank, Washington, D.C.

Franco, Rolando und Martín Hopenhayn (2010). Las clases medias en America Latina: Historias cruzadas y miradas diversas. In: Rolando Franco, Martín Hopenhayn und Arturo León (Hg.). Las clases medias en América Latina: retrospectiva y nuevas tendencias. Mexico City: Siglo Veintiuno, 742.

Franco, Rolando, Martín Hopenhayn und Arturo León (Hg.) (2010). Las clases medias en América Latina: retrospectiva y nuevas tendencias. Mexico City: Siglo Veintiuno.

Gasparini, Leonardo (2003). Income Inequality in Latin America and the Caribbean: Evidence from Household Surveys. Documento de Trabajo Nr. 2. CEDLAS, Centro de Estudios Distributivos, Laborales y Sociales, La Plata. 
Gasparini, Leonardo, Guillermo Cruces, Leopoldo Tornarolli und Mariana Marchionni (2009). A Turning Point? Recent Developments on Inequality in Latin America and the Caribbean. Documento de Trabajo Nr. 81. CEDLAS, Centro de Estudios Distributivos, Laborales y Sociales, La Plata.

Gasparini, Leonardo, Guillermo Cruces und Leopoldo Tornarolli (2011). Recent Trends in Income Inequality in Latin America. Economía, 11(2), 147-201.

Gasparini, Leonardo, Matías Horenstein und Sergio Olivieri (2006). Economic Polarisation in Latin America and the Caribbean: What do Household Surveys Tell Us? Documento de Trabajo Nr. 38. CEDLAS, Centro de Estudios Distributivos, Laborales y Sociales, La Plata.

Gaviria, Alejandro (2007). Social Mobility and Preferences for Redistribution in Latin America. Economía, 8(1), 55-96.

Gayo, Modesto (2013). El problema de la delimitación o boundary problem. Una aproximación a la definición de la clase media. Polis. Revista Latinoamericana, 36, 1-17.

Gilbert, Dennis (2016). The Middle Class: Political, Economic, and Social Perspectives. Latin American Research Review, 51(1), 255-265.

Goldthorpe, John Harry (1985). Soziale Mobilität und Klassenbildung. Zur Erneuerung einer Tradition soziologischer Forschung. In: Hermann Strasser und John Harry Goldthorpe (Hg.). Die Analyse sozialer Ungleichheit. Kontinuität, Erneuerung, Innovation. Wiesbaden: Westdeutscher Verlag, 174-203.

Graciarena, Jorge (1967). Poder y clases sociales en el desarrollo de América Latina. Buenos Aires: Paidós.

Graham, Carol (2002). Mobility, Opportunity and Vulnerability: The Dynamics of Poverty and Inequality in a Global Economy. Journal of Human Development, 3(1), 57-94.

Graham, Carol und Stefano Pettinato (2002). Frustrated Achievers: Winners, Losers and Subjective Well-Being in New Market Economies. The Journal of Development Studies, 38(4), 100-140.

Graham, Carol und Sandip Sukhtankar (2002). Is Economic Crisis Reducing Support for Markets and Democracy in Latin America? Some Evidence from the Economics of Happiness. SSRN Journal.

Grynspan, Rebeca und Ludolfo Paramio (Hg.) (2012). Clases medias en sociedades desiguales. Pensamiento Iberoamericano Nr. 10 (2012/1), AECID, Agencia Española de Cooperación Internacional para el Desarrollo, Madrid.

Harris, Richard L. (2000). The Effects of Globalization and Neoliberalism in Latin America at the Beginning of the Millennium. Journal of Developing Societies, 16(1), 139-162.

Harris, Richard L. (2002). Resistance and Alternatives to Globalization in Latin America and the Caribbean. Latin American Perspectives, 29(6), 136-151.

Hertova, Dagmar, Luis Felipe López-Calva und Eduardo Ortiz-Juárez (2010). Bigger... but Stronger? The Middle Class in Chile and Mexico in the Last Decade. Research for Public Policy. Inclusive Development Nr. ID-02-2010. UNDP, United Nations Development Programme, New York.

Huber, Evelyne (2009). Including the Middle Classes? Latin American Social Policies after the Washington Consensus. In: Monique Kremer, Peter van Lieshout und Robert Went (Hg.). Doing Good or Doing Better. Development Policies in a Globalizing World. Amsterdam: Amsterdam University Press, 137-156. 
IDB, Inter-American Development Bank (1998). Facing Up to Inequality in Latin America: Economic and Social Progress in Latin America. 1998-1999 Report, Washington, D.C.

Jesson, Jill K., Lydia Matheson und Fiona M. Lacey (2011). Doing Your Literature Review. Traditional and Systematic Techniques. Los Angeles: Sage Publications.

Justino, Patricia, Julie Litchfield und Laurence Whitehead (2003). The Impact of Inequality in Latin America. Prus Working Paper Nr. 21. Poverty Research Unit at Sussex, Falmer, Brighton.

Kanbur, Ravi und Nora Lustig (1999). Why is Inequality Back on the Agenda? Paper prepared for the Annual Bank Conference on Development Economics April 28-30, 1999. Draft. The World Bank, Washington, D.C.

Kenny, Charles (2011). The Myth of the Middle Class. Foreign Policy, 19.09.2011. Zugriff am 01.05.2019 auf http://www.foreignpolicy.com/articles/2011/09/19/the myth of the middle class.

Kharas, Homi (2010). The emerging middle class in developing countries. Working Paper Nr. 285. OECD Development Centre, Paris.

Kharas, Homi und Geoffrey Gertz (2010). The New Global Middle Class: A Cross-Over from West to East. Draft version of Chapter 2 in "China's Emerging Middle Class: Beyond Economic Transformation". Wolfensohn Center for Development at Brookings, New York.

Klein, Emilio und Víctor E. Tokman (2000). La estratificación social bajo la tensión en la era de la globalización. Revista de la CEPAL, 72, 7-30.

Krais, Beate und Gunter Gebauer (2002). Habitus. Bielefeld: transcript Verlag.

Kremer, Monique, Peter van Lieshout und Robert Went (Hg.) (2009). Doing Good or Doing Better. Development Policies in a Globalizing World. Amsterdam: Amsterdam University Press.

La Torre, Augusto de, Julian Messina und Joana Silva (2017). The Inequality Story in Latin America and the Caribbean: Searching for an Explanation. In: Luis Bértola und Jeffrey G. Williamson (Hg.). Has Latin American Inequality Changed Direction? Looking Over the Long Run. Cham: Springer, 317-338.

Larrañaga, Osvaldo und María Eugenia Rodríguez (2014). Clases medias y educación en América Latina. Documento de Trabajo. UNDP, United Nations Development Programme, Chile.

Levy, Frank (1987). The Middle Class: Is It Really Vanishing? The Brookings Review, 5(3), 17-21.

López-Calva, Luis Felipe (2013). What Does it Mean to be in the Middle? Mobility and Vulnerability in Latin America and the Caribbean. UNU-WIDERAngle-BLOG. Zugriff am 27.05.2019. www.wider.unu.edu/publication/what-does-it-mean-be-middle.

López-Calva, Luis Felipe, Guillermo Cruces, Samantha Lach und Eduardo Ortiz-Juárez (2014). Clases medias y vulnerabilidad a la pobreza. Reflexiones desde América Latina. El trimestre económico, 81(2), 281-307.

López-Calva, Luis Felipe und Nora Lustig (Hg.) (2010). Declining Inequality in Latin America. A Decade of Progress? Washington, D.C.: Brookings Institution Press.

López-Calva, Luis Felipe und Nora Lustig (2012). El mercado laboral, el Estado y la dinámica de la desigualdad en América Latina: Brasil, México y Uruguay. In: Rebeca Grynspan und Ludolfo Paramio (Hg.). Clases medias en sociedades desiguales. Pensamiento Iberoamericano Nr. 10 (2012/1), AECID, Agencia Española de Cooperación Internacional para el Desarrollo, Madrid, 3-28. 
López-Calva, Luis Felipe und Eduardo Ortiz-Juárez (2011). A Vulnerability Approach to the Definition of the Middle Class. Policy Research Working Paper Nr. 5902. The World Bank, Washington, D.C.

López-Calva, Luis Felipe, Jamele Rigolini und Florencia Torche (2012). Is There Such Thing as Middle Class Values? Class Differences, Values and Political Orientations in Latin America. CGD - Working Paper Nr. 286. CGD, Center for Global Development, Washington, D.C.

Lora, Eduardo A. und Johanna Fajardo (2011). Latin American Middle Classes. The Distance between Perception and Reality. IDB Working Paper Series Nr. 275. IDB, Inter-American Development Bank, Washington, D.C.

Lora, Eduardo A. und Johanna Fajardo (2013). Latin American Middle Classes: The Distance between Perception and Reality. Economía, 14(1), 33-54.

Lustig, Nora (Hg.) (1995). Coping with Austerity. Poverty and Inequality in Latin America. Washington, D.C.: The Brookings Institution.

Lustig, Nora, Luis Felipe López-Calva, Eduardo Ortiz-Juárez und Célestin Monga (2016). Deconstructing the Decline in Inequality in Latin America. In: Kaushik Basu und Joseph Eugene Stiglitz (Hg.). Inequality and Growth. Volume II: Regions and Regularities. London: Palgrave Macmillan UK, 212-247.

Milanovic, Branko (2005). Worlds Apart. Measuring International and Global Inequality. Princeton, Oxford: Princeton University Press.

Milanović, Branko und Shlomo Yitzhaki (2002). Decomposing World Income Distribution: Does the World Have a Middle Class? Review of Income and Wealth, 48(2), 155-178.

Minujin, Alberto (1995). Squeezed: the Middle-Class in Latin America. Environment and Urbanization, $7(2), 153-166$.

Molina, Carlos Gerardo (Hg.) (2006). Universalismo básico: una nueva política social para América Latina. Washington, D.C.: Editorial Planeta.

Morley, Samuel A. (2001). The Income Distribution Problem in Latin America and the Caribbean. Santiago de Chile: United Nations Publications.

Neri, Marcelo (2015). Brazil's New Middle Classes: The Bright Side of the Poor. In: Jeff DaytonJohnson (Hg.). Latin America's Emerging Middle Classes. Economic Perspectives. London: Palgrave Macmillan UK, 70-100.

OECD Development Centre (2010). Latin American Economic Outlook 2011. How Middle-Class Is Latin America? Paris: OECD Publishing.

Oesch, Daniel (2006). Redrawing the Class Map. Stratification and Institutions in Britain, Germany, Sweden and Switzerland. Basingstoke, New York: Palgrave Macmillan.

Olivieri, Sergio (2007). Debilitamiento de la clase media: Gran Buenos Aires 1986-2004. Tesis Maestría en Economía. Facultad de Ciencias Económicas. Universidad Nacional de La Plata, La Plata.

Paes de Barros, Ricardo, Francisco H. G. Ferreira, José R. Molinas Vega und Jaime Saavedra Chanduvi (2009). Measuring Inequality of Opportunities in Latin America and the Caribbean. Washington, D.C.: The World Bank. 
Parker, David Stuart (2012). Introduction: The Making and Endless Remaking of the Middle Class. In: David Stuart Parker und Louise E. Walker (Hg.). Latin America's Middle Class. Unsettled Debates and New Histories. Lanham: Lexington, 1-22.

Penfold, Michael und Guillermo Rodríguez Guzmán (2014). The Growing but Vulnerable Middle Class in Latin America. Growth Patterns, Values and Preferences. Public Policy and Productive Transformation Series Nr. 17. CAF, Development Bank of Latin America, Caracas.

Portes, Alejandro und Kelly Hoffman (2003). Latin American Class Structures. Their Composition and Change during the Neoliberal Era. Latin American Research Review, 38(1), 41-82.

Pressman, Steven (2011). La clase media en países latinoamericanos. Revista Problemas del Desarrollo, 42(164), 127-152.

Psacharopoulos, George, Samuel A. Morley, Ariel Fiszbein, Haeduck Lee und Bill Wood (1997). Poverty and Income Distribution in Latin America. The Story of the 1980s. World Bank Technical Paper Nr. 351. The World Bank, Washington, D.C.

Ravallion, Martin (2009). The Developing World's Bulging (but Vulnerable) "Middle Class". Policy Research Working Paper Nr. 4816. The World Bank, Washington, D.C.

Rehbein, Boike und Jessé Souza (2014). Ungleichheit in kapitalistischen Gesellschaften. Weinheim: Beltz Juventa.

Rigolini, Jamele (2012). Latin America's Middle Class In Global Perspective. Americas Quarterly, 6(4), $58-65$.

Rodrik, Dani (1999). Where Did All the Growth Go? External Shocks, Social Conflict, and Growth Collapses. Journal of Economic Growth, 4(4), 385-412.

Rodrik, Dani (2001). Why Is There so Much Economic Insecurity in Latin America? CEPAL Review, 73, 7-29.

Saavedra, Juan Esteban und Sandra García (2012). Impacts of Conditional Cash Transfer Programs on Educational Outcomes in Developing Countries. A Meta-analysis. RAND Labor and Population Working Paper Nr. WR-921-1. RAND, Research and Development, Santa Monica, California.

Sangmeister, Hartmut (2004). Eine soziale Marktwirtschaft für Lateinamerika. In: Dieter Nohlen und Hartmut Sangmeister (Hg.). Macht, Markt, Meinungen. Demokratie, Wirtschaft und Gesellschaft in Lateinamerika. Wiesbaden: VS Verlag, 111-124.

Sen, Amartya (1983). Poor, Relatively Speaking. Oxford Economic Papers, 35(2), 153-169.

Sen, Amartya (1995). Inequality Reexamined. New York: Russell Sage Foundation.

Solimano, Andrés (Hg.) (1998). Social inequality. Values, Growth, and the State. Ann Arbor: University of Michigan Press.

Solimano, Andrés (2005). Reassessing Social Policies in Latin America. Growth, Middle Classes and Social Rights. CEPAL Review, 87, 45-60.

Solimano, Andrés (Hg.) (2006a). Vanishing Growth in Latin America. The Late Twentieth Century Experience. Cheltenham, U.K, Northampton, Massachusetts: Edward Elgar.

Solimano, Andrés (2006b). Asset Accumulation by the Middle Class and the Poor in Latin America: Political Economy and Governance Dimensions. Serie Macroeconomía del Desarrollo Nr. 55. CEPAL, Comisión Económica para América Latina y el Caribe, Santiago de Chile. 
Solimano, Andrés (2008). The Middle Class and the Development Process. Serie Macroeconomía del Desarrollo Nr. 65. CEPAL, Comisión Económica para América Latina y el Caribe, Santiago de Chile.

Solimano, Andrés (2009). Stylized Facts on the Middle Class and the Development Process. In: Antonio Estache und Danny Leipziger (Hg.). Stuck in the Middle. Is Fiscal Policy Failing the Middle Class? Washington, D.C.: Brookings Institution Press, 24-53.

Solimano, Andrés (2014a). Entrepreneurship, the Middle Class, and Social Mobility: An Overview of Literature. In: Eduardo Lora und Francesca Castellani (Hg.). Entrepreneurship in Latin America. A Step Up the Social Ladder?, Bd. 1. Washington: The World Bank, 17-49.

Solimano, Andrés (2014b). Economic Elites, Crises, and Democracy. Alternatives Beyond Neoliberal Capitalism. Oxford: Oxford University Press.

Solimano, Andrés (2016). Is Inequality Really Declining in Latin America? Evidence on Income, Wealth and the Social Structure. Revista Economica Mundial, 43, 69-92.

Solimano, Andrés und Raimundo Soto (2006). Economic Growth in Latin America in the Late Twentieth Century: Evidence and Interpretation. In: Andrés Solimano (Hg.). Vanishing Growth in Latin America. The Late Twentieth Century Experience. Cheltenham, U.K, Northampton, Massachusetts: Edward Elgar, 11-45.

Solís, Patricio und Marcelo Boado (Hg.) (2016). Y sin embargo se mueve. Estratificación social y movilidad intergeneracional de clase en América Latina. Ciudad de México: El Colegio de México.

Sosa Elízaga, Raquel (2011). Inequality, Exclusion and Poverty in Latin America. The Vast Social Debt of Neoliberalism. In: Boike Rehbein (Hg.). Globalization and Inequality in Emerging Societies. London: Palgrave Macmillan, 50-70.

Souza, Jessé (2010). Os batalhadores: nova classe média ou classe média trabalhadora. Belo Horizonte: Editora UFMG.

Stampini, Marco, Marcos Robles, Mayra Sáenz, Pablo Ibarrarán und Nadin Medellín (2016). Poverty, Vulnerability, and the Middle Class in Latin America. Latin American Economic Review, 25(4), 144.

Székely, Miguel (2003). The 1990s in Latin America. Another Decade of Persistent Inequality, but with Somewhat Lower Powerty. Journal of Applied Economics, 6(2), 317-339.

Székely, Miguel und Marianne Hilgert (2002). Inequality in Latin America during the 1990s. In: Richard Barry Freeman (Hg.). Inequality Around the World. Basingstoke: Palgrave Macmillan UK, $128-161$.

IBRD, The International Bank for Reconstruction and Development und The World Bank (2007). Global Economic Prospects. Managing the Next Wave of Globalization. Washington, D.C.: The World Bank.

Thompson, Edward Palmer (1963). The Making of the English Working Class. Harmondsworth: Penguin Books.

Thurow, Lester (1987). A Surge in Inequality. Scientific American, 256(5), 30-37.

Torche, Florencia (2005). Unequal but Fluid: Social Mobility in Chile in Comparative Perspective. American Sociological Review, 70(3), 422-450. 
Torche, Florencia (2006). Una clasificación de clases para la sociedad chilena. Revista de sociología, 20, $15-43$.

Torche, Florencia und Luis Felipe López-Calva (2012). Stability and Vulnerability of the Latin American Middle Class. UNU WIDER Working Paper Nr. 2012/98. UNU-WIDER - World Institute for Development Economic Research, Helsinki.

Tornarolli, Leopoldo (2014). The Evolution of the Middle Class in Latin America. Policy Brief Nr. 45 (July). International Policy Center for Inclusive Growth, Brasilia.

UNDP, United Nations Development Programme (2014). Perfil de estratos sociales en América Latina: pobres, vulnerables y clases medias, New York.

Vester, Michael, Peter von Oertzen, Heiko Geiling, Thomas Hermann und Dagmar Müller (2001). Soziale Milieus im gesellschaftlichen Strukturwandel. Zwischen Integration und Ausgrenzung. Frankfurt a. M.: Suhrkamp.

Wacquant, Löic (1991). Making Class: the Middle Class(es) in Social Theory and Social Structure. In: Scott G. MacNall, F. Levine Rhonda und Rick Fantasia (Hg.). Bringing Class Back in Contemporary and Historical Perspectives. Boulder: Westview Press, 39-64.

Wehr, Ingrid (2011). Zur problematischen Koexistenz von Demokratie und eklatanter sozialer Ungleichheit in Lateinamerika. In: Ingrid Wehr und Hans-Jürgen Burchardt (Hg.). Soziale Ungleichheiten in Lateinamerika. Neue Perspektiven auf Wirtschaft, Politik und Umwelt. Baden-Baden: Nomos, 9-28.

Williamson, John (1990). What Washington means by policy reform. In: John Williamson (Hg.). Latin American adjustment. How much has happened? Washington, D.C.: Peterson Institute for International Economics, 90-120.

Wilson, Dominic und Raluca Dragusanu (2008). The Expanding Middle: The Exploding World Middle Class and Falling Global Inequality. Global Economics Paper Nr. 170. Goldman Sachs, New York.

Wright, Eric Olin (1985b). Wo liegt die Mitte der Mittelklasse? PROKLA. Zeitschrift für kritische Sozialwissenschaft, 58, 35-62.

Zhu, Feng (2005). A Nonparametric Analysis of the Shape Dynamics of the US Personal Income Distribution: 1962-2000. BIS Working Papers Nr. 184. Bank for International Settlements, Basel. 